\title{
Activity-induced synaptic delivery of the GluN2A-containing NMDA receptor is dependent on endoplasmic reticulum chaperone Bip and involved in fear memory
}

Xiao-min Zhang ${ }^{1,2}$, Xun-yi Yan ${ }^{1}$, Bin Zhang ${ }^{1}$, Qian Yang ${ }^{1}$, Mao Ye ${ }^{1}$, Wei Cao ${ }^{1}$, Wen-bin Qiang ${ }^{1}$, Li-jun Zhu ${ }^{1}$, Yong-lan $\mathrm{Du}^{1}$, Xing-xing Xu ${ }^{1}$, Jia-sheng Wang ${ }^{1}$, Fei Xu${ }^{1}$, Wei Lu ${ }^{3}$, Shuang Qiu ${ }^{1}$, Wei Yang ${ }^{1}$, Jian-hong Luo ${ }^{1}$

${ }^{I}$ Department of Neurobiology, Key Laboratory of Medical Neurobiology (Ministry of Health of China), Collaborative Innovation Center for Brain Science, Zhejiang University School of Medicine, Hangzhou, Zhejiang 310058, China; ${ }^{2}$ Department of Physiology, Kunming Medical University, Kunming, Yunnan 650500, China, ${ }^{3}$ Key Laboratory of Developmental Genes and Human Disease (Ministry of Education of China), Institute of Life Sciences, Southeast University, Nanjing, Jiangsu 211189, China

The N-methyl-D-aspartate receptor (NMDAR) in adult forebrain is a heterotetramer mainly composed of two GluN1 subunits and two GluN2A and/or GluN2B subunits. The synaptic expression and relative numbers of GluN2Aand GluN2B-containing NMDARs play critical roles in controlling $\mathrm{Ca}^{2+}$-dependent signaling and synaptic plasticity. Previous studies have suggested that the synaptic trafficking of NMDAR subtypes is differentially regulated, but the precise molecular mechanism is not yet clear. In this study, we demonstrated that Bip, an endoplasmic reticulum (ER) chaperone, selectively interacted with GluN2A and mediated the neuronal activity-induced assembly and synaptic incorporation of the GluN2A-containing NMDAR from dendritic ER. Furthermore, the GluN2A-specific synaptic trafficking was effectively disrupted by peptides interrupting the interaction between Bip and GluN2A. Interestingly, fear conditioning in mice was disrupted by intraperitoneal injection of the interfering peptide before training. In summary, we have uncovered a novel mechanism for the activity-dependent supply of synaptic GluN2A-containing NMDARs, and demonstrated its relevance to memory formation.

Keywords: NMDA receptors; endoplasmic reticulum; Bip; non-canonical trafficking; fear conditioning Cell Research (2015) 25:818-836. doi:10.1038/cr.2015.75; published online 19 June 2015

\section{Introduction}

The N-methyl-D-aspartate receptor (NMDAR) plays vital roles during brain development, plasticity, and pathology. Functional NMDARs are tetramers, mainly composed of two obligatory GluN1 subunits and two regulatory GluN2 subunits. The GluN2A and GluN2B subunits are prominently expressed in the forebrain and endow the NMDARs with distinct functional properties in terms of channel kinetics, expression pattern, and coupling to intracellular signaling [1-2]. Importantly, the number and

Correspondence: Jian-hong Luo ${ }^{\mathrm{a}}$, Wei Yang ${ }^{\mathrm{b}}$, Shuang Qiu

${ }^{a}$ E-mail: luojianhong@zju.edu.cn

${ }^{b} E$-mail: yangwei@zju.edu.cn

'E-mail: qiushly@zju.edu.cn

Received 8 October 2014; revised 2 March 2015; accepted 4 May 2015; published online 19 June 2015 composition of the GluN2A- and GluN2B-containing NMDARs on synapses are dynamically regulated during development and neuronal activation, and this is thought to govern synaptic plasticity. A switch in the composition of synaptic NMDARs from GluN2B to GluN2A during development has been extensively studied [3-5]. An increase of synaptic GluN2A-containing NMDARs has also been found in adult mice after environmental stimulation [6-10] and even after behavioral training [11-12].

Interestingly, the neuronal activity-induced regulation of synaptic GluN2A- and GluN2B-containing NMDARs does not run in parallel. The rapid upregulation of GluN2A-containing NMDARs is not usually accompanied by an increase of those containing GluN2B, suggesting that the two subtypes are regulated through differential but coordinated trafficking pathways. The surface expression of both GluN2A and GluN2B can be regulated by phosphorylation [13]. The phosphorylation of GluN2B 
results in a change of its lateral diffusion between synaptic and extrasynaptic regions and its endocytosis [14-17]. It has been suggested that the endocytosis of GluN2A can also be regulated by phosphorylation [18]. However, GluN2A is less mobile than GluN2B and is more stably located at the synapse, thus its surface expression may be regulated by other process. Current evidence [19-20] indicates that there are two protein-trafficking modalities in dendrites. In the canonical pathway, the synthesis and maturation of nascent proteins are completed in the soma and then these proteins are transported to distal dendrites via Golgi-sorted vesicles. In the non-canonical pathway, proteins bypass the somatic Golgi apparatus and are transported to dendritic Golgi outposts via the dendritic ER $[19,20]$. It was earlier believed that the GluN2B subunit is transported from soma to distal dendrite in the form of Golgi-sorted vesicles and depends on a neuron-specific kinesin, kif17 [21, 22], suggesting that GluN2B is transported via the canonical trafficking pathway. However, a recent study has indicated that the GluN1 and GluN2B subunits can be transported via a non-canonical pathway in which they bypass the somatic Golgi and are transported along dendrites within the ER, and this process is mediated by SAP97 and CASK [23].

Less is known about the precise mechanism of GluN2A trafficking. Existing evidence suggests that GluN2A is likely transported via a non-canonical pathway [24, 25]. It has been reported that SAP97, which mediates the non-canonical trafficking of GluN2B and GluN1, also interacts with GluN2A within the ER and their interaction can be regulated by CaMKII [24]. Furthermore, it was recently found that dendritic synthesis of GluN2A is initiated after the induction of chemical long-term potentiation (cLTP) $[25,26]$. This evidence indicates that GluN2A can be transported directly from dendrite into synapse without lateral trafficking, thus independently of kinesin and microtubules. This trafficking feature of GluN2A differs from that of GluN1 and GluN2B, which require the microtubule-dependent motor protein kif17 even for non-canonical trafficking. Nevertheless, more evidence is required to better understand the molecular mechanism of the non-canonical trafficking of GluN2A during neuronal activation and its significance. In this study, we uncovered a novel mechanism for the synaptic delivery of the GluN2A-containing NMDAR from the dendritic ER. We identified Bip, an ER chaperone, as a selective GluN2A-binding protein and a mediator in the assembly and delivery of the GluN2A-containing NMDAR to the synapse during neuronal activation. Furthermore, we found that interrupting the binding of GluN2A to Bip effectively impaired the acquisition of fear conditioning in mice.

\section{Results}

ER chaperone Bip selectively interacts with the GluN2A subunit of NMDAR

In a previous study, we found an ER retention signal located in the amino-terminal domain (ATD) of GluN2A, but not its homolog GluN2B [27]. Thus, we set out to further explore the molecular mechanism underlying this signal-mediated GluN2A ER retention and its function. We started by screening the proteins that interacted with the ATD of GluN2A. GFP-tagged GluN2A-ATD was expressed in HEK293 cells transfected with pD-ATD ${ }_{2 A^{-}}$ GFP. This is a single transmembrane domain protein facing ER luminal side. The cell lysate was co-immunoprecipitated with GFP antibodies, and the specific bands were analyzed by mass spectrometry. We therefore identified a GluN2A-ATD-interacting ER-located chaperone protein, Bip (Figure 1A), and confirmed that Bip selectively interacted with the ATD of GluN2A but not that of GluN2B (Figure 1B). We further confirmed that Bip did not interact with GluN1 or GluN2B in rat cortical tissue at embryonic day 18 (E18), when Glu$\mathrm{N} 2 \mathrm{~A}$ is not yet expressed, while in the adult rat cortex Bip had a much stronger interaction with GluN2A than with GluN2B or GluN1 (Figure 1C). The interaction between the substrate-binding domain (SBD) of Bip and GluN2A was further examined by GST pull-down assay (Figure 1D). Using co-immunoprecipitation (coIP) and mass-spectrometry, we also identified two other ER chaperones, GRP94 and ERp72, and found that they interacted with GluN2A-ATD at trace levels in HEK293 cells (Figure 1A and 1B). However, we failed to immunoprecipitate subunits of the NMDAR using antibodies against GRP94 or ERP72, suggesting a weak or unstable interaction between them. Thus, we focused on studying the function of Bip in the trafficking of the GluN2A-containing NMDAR. We examined the distribution of Bip in neurons, and found that it was strongly expressed in both the somata and processes of neurons in the cortex and hippocampus (Figure 1E-1G), and Bip partly co-localized with GluN2A in the dendrites of cultured cortical neurons. However, we did not find co-localization of Bip and PSD95. To further confirm this result, we used Stochastic Optical Reconstruction Microscopy (STORM) to analyze the sub-localization of Bip in dendrites at higher spatial resolution [28], and found that Bip was mainly expressed in the dendritic shaft but rarely co-localized with PSD95 (Figure 1H). Consistent with this, Bip was absent from the Triton X-insoluble postsynaptic density (PSD) fraction (Figure 1I). These data showed that the ER chaperone, Bip, binds specifically with GluN2A through its SBD domain, suggesting that Bip might se- 
A

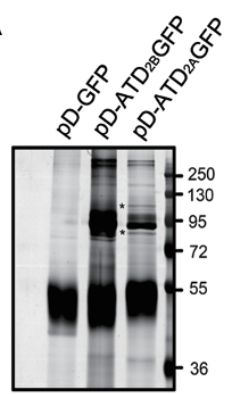

B

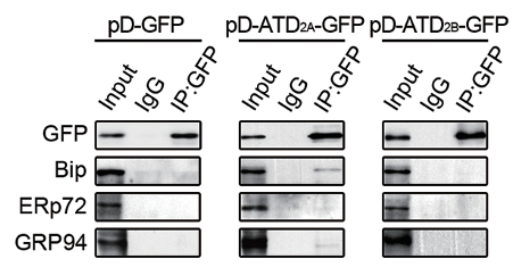

D
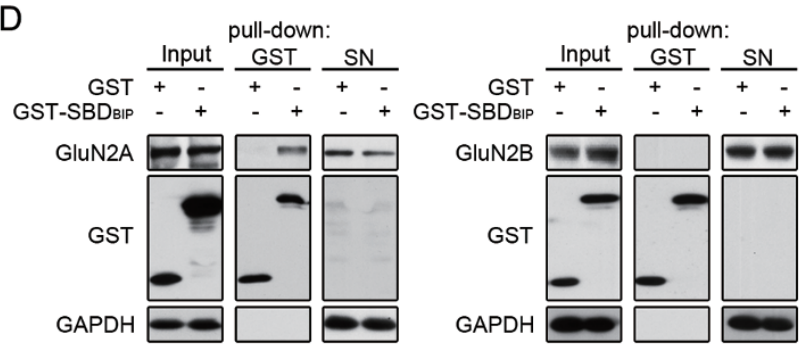

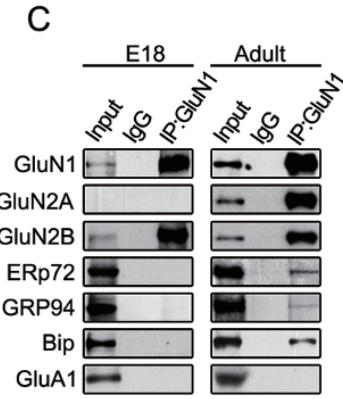

E

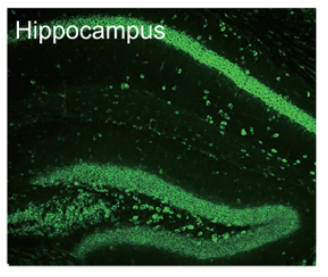

F
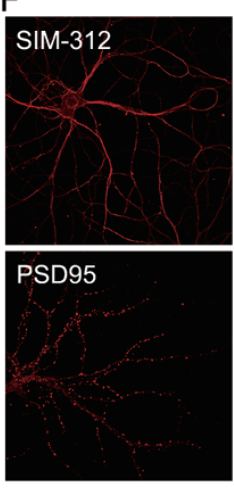
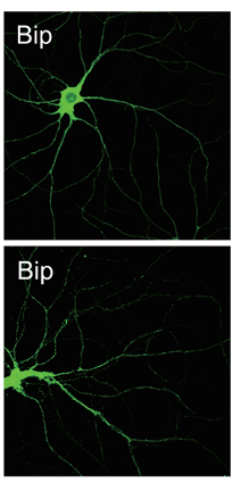

G

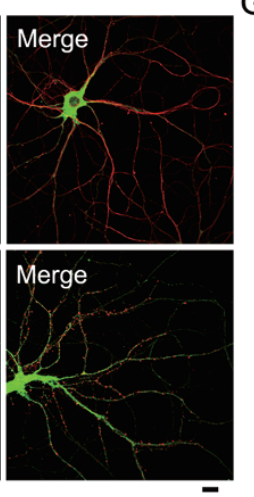

\begin{tabular}{|l|l|}
\hline SIM-312 & Bip \\
\hline PSD95 & Bip \\
\hline GluN2A & Bip \\
\hline GluN2B & Bip \\
\hline
\end{tabular}

\begin{tabular}{|l|}
\hline Merge \\
\hline Merge \\
\hline Merge \\
\hline Merge \\
\hline
\end{tabular}

$\mathrm{H}$
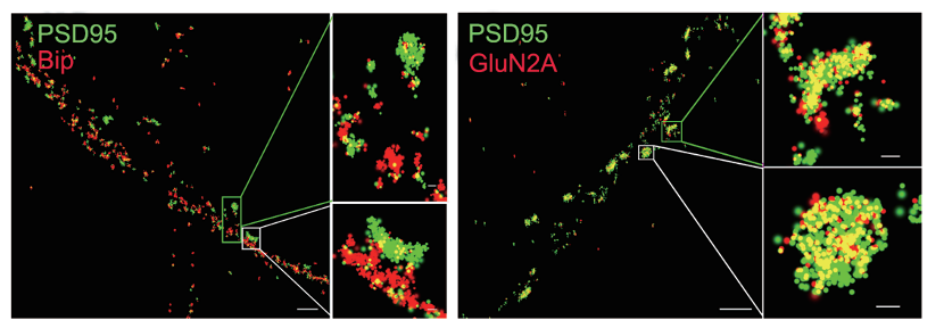

I

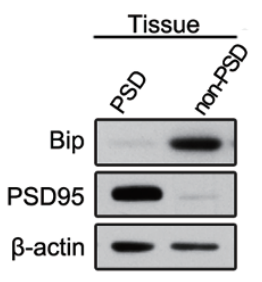

Figure 1 ER chaperone Bip specifically binds to the GluN2A subunit. (A) The lysates from pD-ATD $2 A-G F P-, p D-A T D_{2 B}-G F P-$ and pD-GFP-transfected HEK293 cells were each immunoprecipitated with rabbit anti-GFP and subjected to SDS-PAGE and silver staining. Asterisks indicate two specific bands on representative silver-staining in the pD-ATD ${ }_{2 A}-G F P$ column $(n=10)$. (B) The immunoprecipitate with anti-GFP was analyzed by western blot. Bip interacted specifically with pD-ATD ${ }_{2 A}-$ GFP $(n=$ 3). (C) The interactions between Bip and NMDAR subunits were examined in E18 and adult rat cortex. GluN1 interaction with GluN2A, GluN2B, ERp72, GRP94, or Bip was assessed by co-IP using GluN1 antibody (left). Bip interaction with GluN2A, GluN2B, GluN1 or GRP94 was assessed by co-IP using Bip antibody (right) $(n=7)$. (D) Direct interaction between the substrate-binding domain (SBD) of Bip and GluN2A or GluN2B detected by GST pull-down assay. The SBD of Bip was fused with GST to make the chimera protein GST-SBD Bip. GST and GST-SBD Bip $_{\text {w }}$ were expressed in E. coli and used to pull down GluN2A or GluN2B from adult rat cortex lysate. Input, total amount of proteins; GST, proteins pulled down by GST or GST-SBD $D_{\text {Bip }}$ SN, supernatant of the pull-down assay. GAPDH was used as negative control $(n=3)$. (E) Expression pattern of Bip in adult rat hippocampus and cortex, shown by immunohistochemistry. Scale bar $=200 \mu \mathrm{m}$. (F, G) Expression pattern of Bip in cultured cortical neurons shown by immunocytochemistry. Scale bars $=10 \mu \mathrm{m}$. $(\mathrm{H})$ Localization of Bip (red) and GluN2A (red) with PSD95 (green) were each assessed by STORM. (I) The Triton X-insoluble PSD fraction was extracted and the expression of Bip in the PSD fraction was assessed. 
lectively participate in regulating the trafficking of the GluN2A- but not the GluN2B-containing NMDAR.

Synaptic expression of the GluN2A-containing NMDAR increases after cLTP induction

Next, we further explored the potential role of Bip in the dendritic trafficking of GluN2A. We induced cLTP in cultured neurons since it has been reported that the surface expression of the GluN2A-containing NMDAR increases in this condition $[25,29]$. By using surface biotinylation, we found that indeed the surface expression of GluN2A increased after cLTP induction, while that of GluN2B decreased (Figure 2A and 2B). We then assessed the accumulation of GluN2A in the Triton $\mathrm{X}$-insoluble PSD fraction with the same stimulation, and found that it also increased, while GluN2B decreased (Figure 2C and 2D). To further confirm these results, we assessed the surface expression of transfected CFP-GluN2A and CFP-GluN2B in cultured cortical neurons and obtained consistent results (Figure 2E and 2F). To ensure the effectiveness of the cLTP stimulation in our experiments, the phosphorylation of GluA1 serine 845 of the $\alpha$-amino-3-hydroxy-5-methyl-4-isoxazolepropionic acid receptor (AMPAR) was assessed and it was increased as predicted (Figure 2C). We further found that under the same conditions both the surface expression and the PSD accumulation of GluA1 increased (Figure 2A-2F), as predicted [29-30]. These results are consistent with previous studies showing that the cLTP protocol effectively increases the surface expression of the GluN2A-containing NMDAR. In addition, the increase of GluN2A in the PSD fraction suggests a synaptic accumulation of the GluN2A-containing NMDAR.

Bip participates in the synaptic insertion of the GluN2A-containing NMDAR during cLTP

To further explore the role of Bip in the surface expression of the GluN2A-containing NMDAR, we investigated the change of the interaction between Bip and GluN2A by co-IP after induction of cLTP. The results showed that the fraction of GluN2A binding with Bip declined, while the interaction between Bip and GRP94 was not affected (Figure 3A and 3C). Meanwhile, the fraction of GluN2A associated with GluN1 increased (Figure 3B and 3C). However, it is possible that this change was a natural result of forward-trafficking of Glu$\mathrm{N} 2 \mathrm{~A}$. To determine whether the interaction between Bip and GluN2A was dynamically modulated by neuronal activity, we used Brefeldin A (BFA; $20 \mu \mathrm{g} / \mathrm{ml}$ ) [31] to inhibit the forward transport of GluN2A. BFA inhibits the transport of nascent proteins by preventing the formation of coat protein I vesicles, and as a result the nascent pro- teins accumulate in the ER [32]. If the separation of Bip and GluN2A was simply a result of forward transport of GluN2A, it would be prevented by BFA. However, we found that BFA did not inhibit the separation of GluN2A from Bip (Figure 3A and 3C). Therefore, the separation induced by cLTP occurred before the transport of GluN2A from ER-to-Golgi, and was not simply caused by forward trafficking. These data also showed that the assembly of GluN1 and GluN2A occurred along with the separation of Bip and GluN2A, suggesting continuity between these two events.

To further characterize the separation of Bip and GluN2A during cLTP, we investigated the change of co-localization between Bip and GluN2A in dendrites. We found that $57.9 \% \pm 1.74 \%(n=18)$ of GluN2A co-localized with Bip in secondary and tertiary dendrites, and the fraction declined significantly after cLTP, suggesting a separation of GluN2A from Bip (Figure 3D and 3E). At the same time, we found that the co-localization of GluN2A and PSD95 increased after cLTP stimulation (Figure $3 \mathrm{D}$ and $3 \mathrm{~F}$ ). The fraction of GluA1 that co-localized with PSD95 also increased, which validated the effectiveness of the stimulation (Figure 3D and 3F) [33]. These data suggested that the release of GluN2A from Bip occurs in dendrites, consistent with a previous study showing that cLTP results in the dendritic synthesis of GluN2A [25]. Together, these data showed that the interaction between Bip and GluN2A was dynamically regulated by cLTP inside the dendrites.

Synaptic increase of the GluN2A-containing NMDAR depends on its trafficking from dendritic ER to synapse

Unlike other cells, neurons have evolved a non-canonical trafficking pathway in which proteins are synthesized and sorted by axonal or dendritic ER and Golgi outposts and transported directly into synapses [19, 20, 34-36]. This pathway is widely recognized for the GABA receptor, the Kv4 $\mathrm{K}^{+}$channel, and the NMDAR [37-39]. It has been reported that the surface expression of GluN2A induced by cLTP depends on dendritic synthesis [25], and this is consistent with our results that GluN2A partly co-localized with the ER chaperone Bip in dendrites and this interaction was dynamically regulated by neuronal activity. However, we did not see a significant change in the total expression of GluN2A during cLTP, which suggested that there may be a reserve pool of GluN2A subunit protein in the dendritic ER for rapid replenishment of synaptic GluN2A-containing NMDARs during cLTP. Thus, we used a set of pharmacological agents to determine whether the GluN2A-containing NMDARs expressed during cLTP induction were transported in through non-canonical pathway (Supplementary infor- 
A

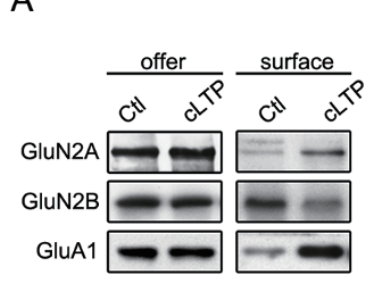

B

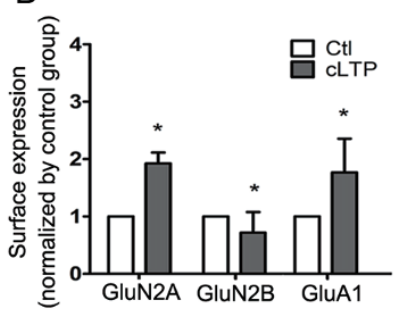

C

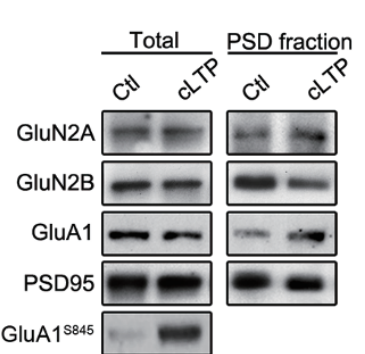

$\mathrm{D}$

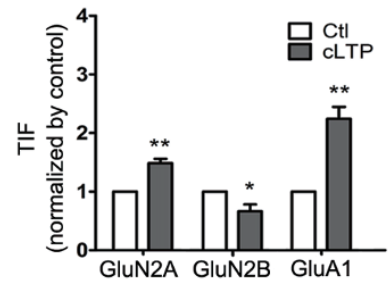

$\mathrm{E}$

CFP-GluN2A
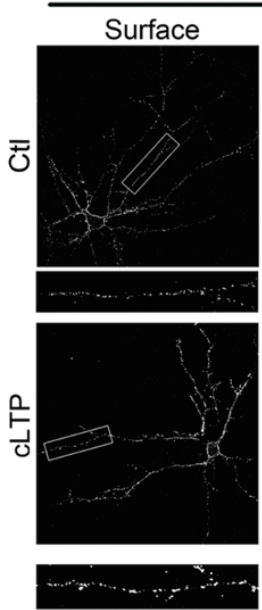
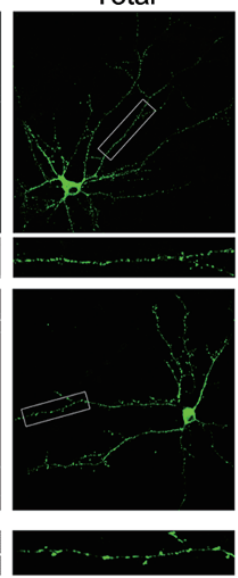
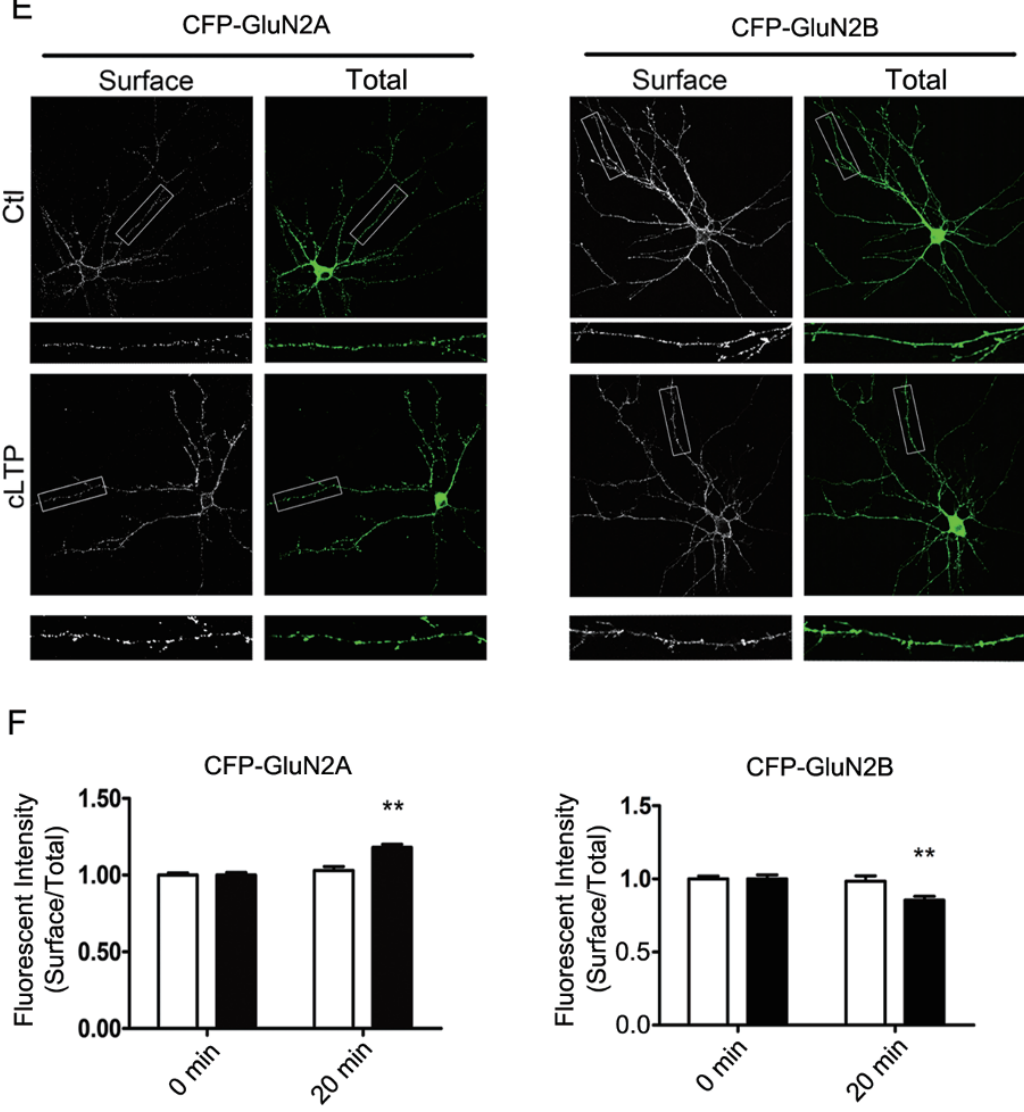

CFP-GluN2B

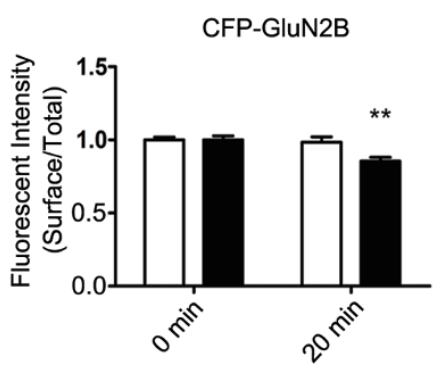

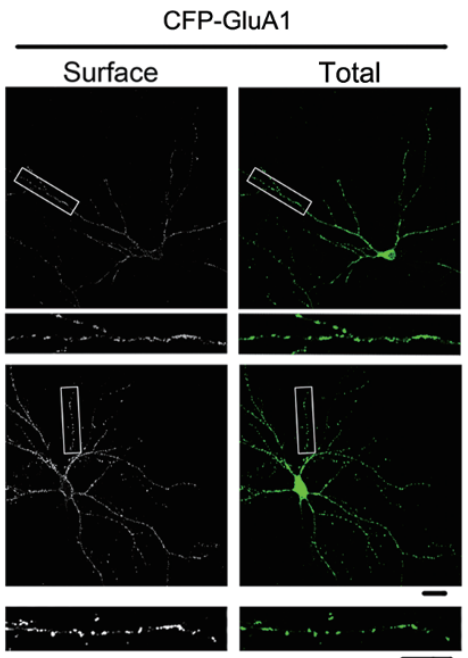

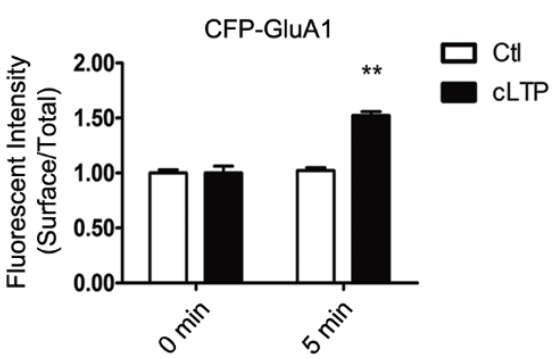

Figure 2 Neuronal activity increases the surface expression of GluN2A-containing NMDARs. (A) The surface expression of endogenous GluN2A, GluN2B, and GluA1 during CLTP was assessed by biotinylation. (B) Quantification of surface expression of endogenous receptors after cLTP. GluN2A: $192.6 \% \pm 18.8 \%, n=5$; GluN2B: $71.7 \% \pm 35.9 \%, n=3$; GluA1: $176.7 \%$ $\pm 58.7 \%, n=3$. (C) The Triton X-insoluble PSD fraction (TIF) was extracted and the increase of endogenous GluN2A and GluA1 in the TIF 20 min after cLTP was quantified. The phosphorylation of serine 845 (GluA1S ${ }^{845}$ ) was also assessed. (D) Quantification of accumulation in the TIF. GluN2A: $148.5 \% \pm 7.5 \%, n=6$; GluN2B: $66.4 \% \pm 11.8 \%, n=6$; GluA1: $224.3 \% \pm$ $20.4 \%, n=5$. (E) CFP-GluN2A, CFP-GluN2B or CFP-GluA1 was transfected into cultured cortical neurons at DIV 10, and surface expression was measured at DIV 14-16. Surface CFP-GluN2A, CFP-GluN2B and CFP-GluA1 were stained with rabbit anti-GFP primary antibody and Alexa 546-conjugated donkey anti-rabbit secondary antibody. The total CFP-GluN2A, CFP-GluN2B and CFP-GluA1 were stained with the same primary antibody but with Alexa 488-conjugated secondary antibody after membrane permeabilization. Scale bar $=50 \mu \mathrm{m}$. (F) Quantification of surface expression of transfected receptors. GluN2A: $118.1 \% \pm 2.01 \%, n=31$; GluN2B: $85.4 \% \pm 2.7 \%, n=31$; GluA1: $152.3 \% \pm 3.5 \%, n=31$. All data are presented as mean \pm SEM. ${ }^{*} P<0.05,{ }^{*} P<0.01$.

mation, Figure S1).

First, we determined whether inhibiting ER-to-Golgi transport abolishes the increase of surface NMDARs by using BFA $(20 \mu \mathrm{g} / \mathrm{ml})$, and 1,3-cyclohexanebis (methylamine) (CBM, $2 \mathrm{mM}$ ), both of which interfere with the early forward transport of integral protein from the ER 
[40]. We applied BFA or CBM simultaneously with cLTP induction and found that both inhibitors blocked the surface increase of CFP-GluN2A in transfected cultured cortical neurons (Figure 4A and 4B). However, it has been reported that high levels of BFA can affect the overall intracellular transport system, including the mobility of endosomes and lysosomes [32]. To exclude this possibility, we assessed whether the surface expression of GluA1 induced by cLTP was inhibited by BFA or CBM, because AMPARs are stored inside synapses in recycling vesicles and their insertion into the synaptic membrane during cLTP is independent of ER-to-Golgi trafficking. As predicted, we found that neither BFA nor CBM inhibited the cLTP-induced surface insertion of CFP-GluA1 (Figure 4C and 4D), suggesting that BFA and CBM did not disturb the exocytosis of AMPARs from the vesicle pool in a nonspecific manner.

We further analyzed GluN2A trafficking during cLTP
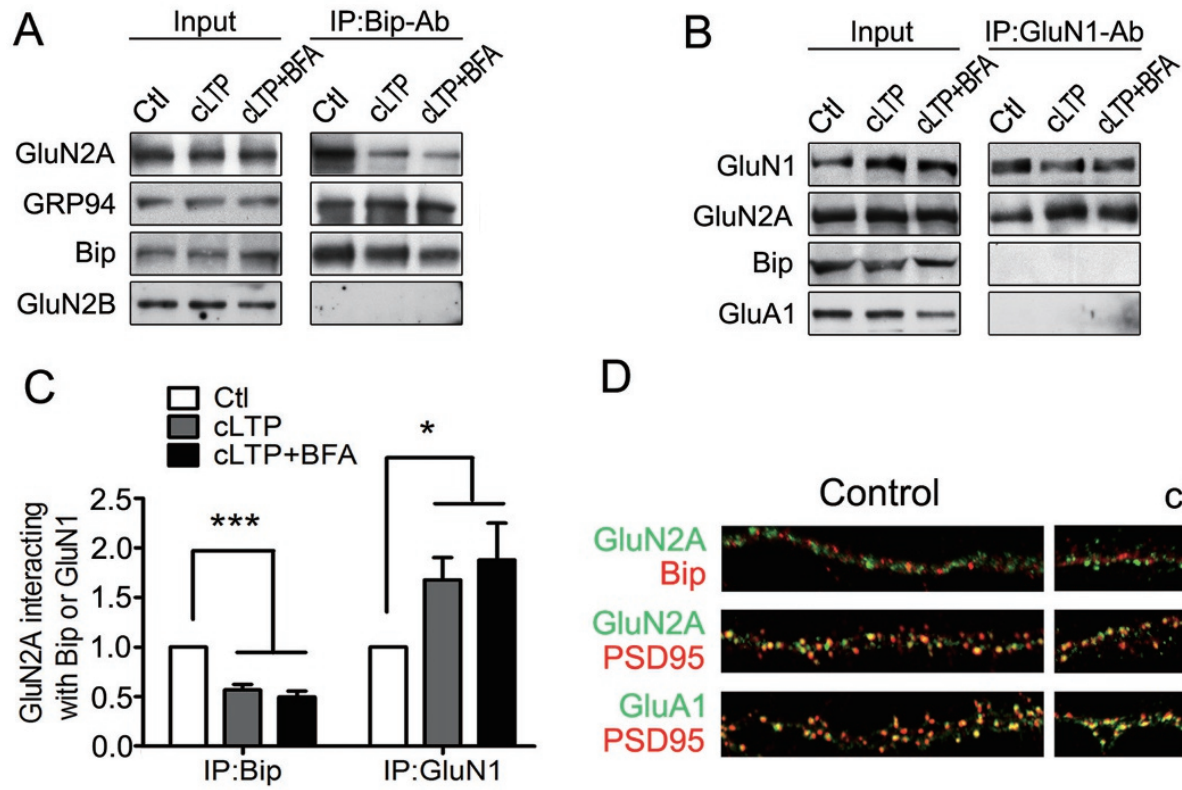

D
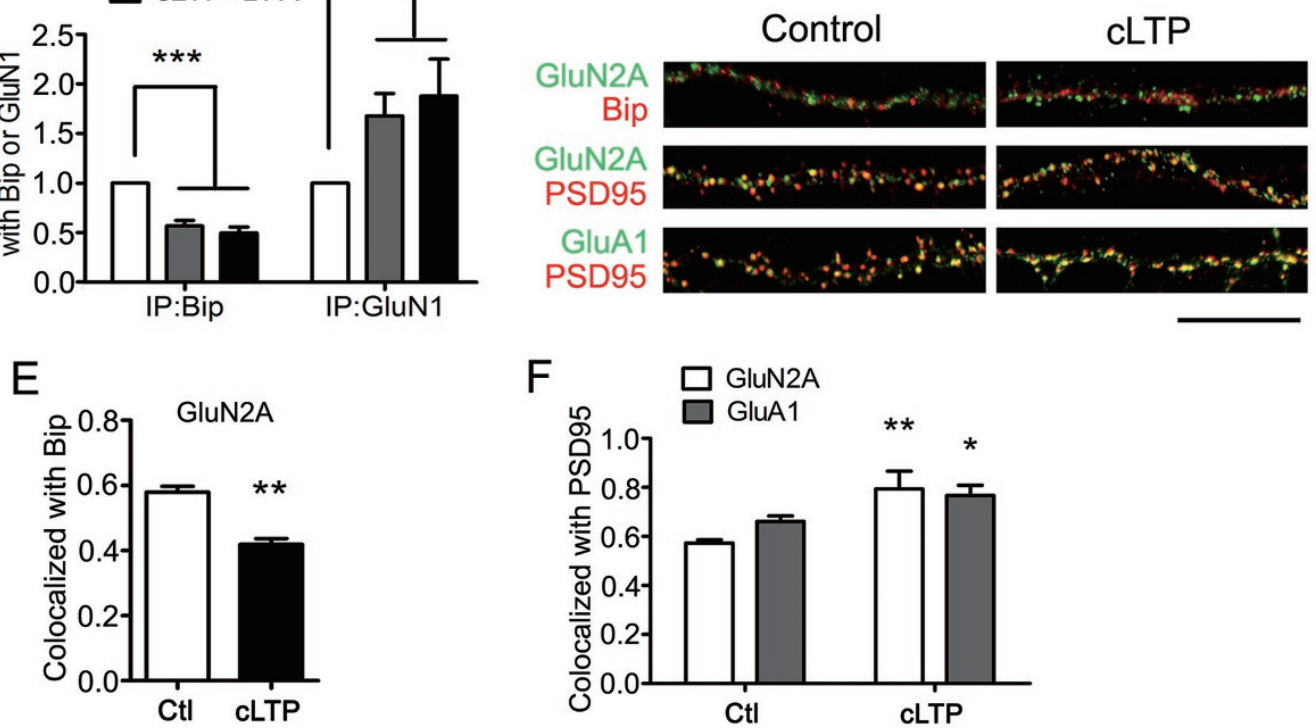

Figure 3 Bip is involved in the activity-dependent surface expression of GluN2A-containing NMDARs. (A) Rabbit anti-Bip antibody was used for co-immunoprecipitation after incubation of cultured cortical neurons in ECS (CtI), and after CLTP (cLTP) or CLTP with BFA (cLTP+BFA). Input: total amount of proteins; IP:Bip-Ab: fraction co-immunoprecipitated by Bip. (B) The GluN1 antibody was used to co-immunoprecipitate GluN2A or Bip from cultured cortical neurons (IP:GluN1-Ab: fraction co-immunoprecipitated by GluN1). (C) Quantification of Bip-GluN2A and GluN1-GluN2A interactions normalized to the control group (Ctl). After cLTP: GluN2A-Bip interaction: 56.7\% $\pm 5.7 \%, n=5$; GluN2A-GluN1 interaction: $167.9 \% \pm 22.4 \%, n=6$. After cLTP with BFA: GluN2A-Bip interaction: $49.7 \% \pm 6.0 \%, n=5$; GluN2A-GluN1 interaction: $188.9 \% \pm 37.3 \%, n=6$. (D) Representative images of co-localization of GluN2A with Bip and PSD95 before and after CLTP stimulation. CFP-GluN2A was first transfected into cultured cortical neurons, and the co-localization of GluN2A with Bip or PSD95 was assessed by primary antibodies against GFP, Bip or PSD95. Scale bar $=10 \mu \mathrm{m}$. (E) Quantification of co-localization between Bip and GluN2A. Before cLTP (CtI): $57.9 \% \pm 1.8 \%, n=18$; after cLTP: $41.9 \% \pm 1.7 \%, n=18$. ${ }^{*} P<0.05$. (F) Quantification of co-localization between GluA1 and GluN2A with PSD95. Before cLTP: GluN2A co-localization with PSD95: $57.3 \% \pm 1.3 \%, n=21$; GluA1 co-localization with PSD95: $66.1 \% \pm 2.3 \%, n=21$. After cLTP: GluN2A co-localization with PSD95: 79.4\% $\pm 7.3 \%, n=21 ;$ GluA1 co-localization with PSD95: $76.7 \% \pm 4.2 \%, n=20$. All data are presented as mean \pm SEM. ${ }^{*} P<0.05,{ }^{* \star} P<0.01,{ }^{* \star *} P<0.001$. 
in a well-established, more physiological model [41, 42], using whole-cell recordings from CA1 pyramidal neurons in hippocampal slices from P7-P12 rats. We delivered an cLTP induction protocol to drive the synaptic GluN2B-to-GluN2A switch in these neurons. After induction and a short stable recording of NMDAR current, ifenprodil $(3 \mu \mathrm{M})$ was added for $>25$ min to block the GluN2B-containing NMDAR current, and the residual current indicated the proportion of GluN2A-containing NMDARs. The residual current was $70 \%$ of the total current after induction, while it was $40 \%$ in the non-induced group (Figure 4E and 4F). This experiment demonstrated that we successfully recorded an increase of GluN2A surface expression after the cLTP induction protocol was delivered. We tested whether the increase was inhibited by BFA, and as predicted we found that intracellular application of BFA attenuated the switch from GluN2B to GluN2A (Figure 4G and 4H). These data showed that the increase of surface GluN2A depended on ER-to-Golgi trafficking, which suggested that the increased GluN2A is delivered to the synaptic surface from the ER. Next, we tested whether nascent protein synthesis is required for the neuronal activity-induced surface expression of GluN2A-containing NMDARs. We found that simultaneous application of the translation inhibitor cycloheximide (CHX, $5 \mu \mathrm{g} / \mathrm{ml}$ ) with cLTP induction was ineffective, but pre-incubation with $\mathrm{CHX}$ for $0.5 \mathrm{~h}$ blocked the surface increase of CFP-GluN2A, and pretreatment with CHX for $1.5 \mathrm{~h}$ failed to inhibit the membrane insertion of AMPARs during cLTP (Supplementary information, Figure S2). Since our construct of CFP-GluN2A contained the 3'UTR which is needed for its dendritic synthesis, these results demonstrated that, consistent with previous studies, the translation of GluN2A was initiated by cLTP [25], and also showed that the protein synthesis and trafficking may be temporally coordinated; that is, trafficking of the receptors is required for rapid insertion, while synthesis serves as a further support and provides a supply for replenishment of the receptor in the dendritic ER.

We further clarified whether the increased surface GluN2A during cLTP was transported via the canonical or the non-canonical pathway. We assessed whether the surface increase of the GluN2A-containing NMDAR depended on microtubules and/or actin, since in canonical trafficking, the transport of nascent protein from soma to distal dendrites requires microtubules, but in non-canonical trafficking the nascent protein is locally synthesized in the ER $[25,26]$ and transport from the dendritic ER into the spine and synapse only requires actin [43]. Thus, if GluN2A was transported through the canonical pathway, the synaptic insertion of GluN2A would be blocked by both the actin inhibitor cytochalasin $\mathrm{D}$ and the mi- crotubule inhibitor nocodazole, but if it was transported through the non-canonical pathway, its synaptic insertion would be insensitive to nocodazole. We first assessed this method by examining the dependence of membrane expression of GluA1 on cytochalasin D and Nocodazole, since AMPARs are stored in recycling endosomes inside the synapse, therefore its membrane expression during LTP is not dependent on microtubules [44]. We incubated cultured cortical neurons with cytochalasin $\mathrm{D}(10 \mu \mathrm{M})$ or Nocodazole $(10 \mu \mathrm{g} / \mathrm{ml})$ for $2 \mathrm{~h}$ to inhibit the actin or microtubules, and found that the increase of surface CFP-GluA1 was abolished by cytochalasin $\mathrm{D}$ but not nocodazole (Figure $5 \mathrm{~A}$ and $5 \mathrm{~B}$ ). We assessed the effects of both drugs on CFP-GluN2A and found that the increase of surface CFP-GluN2A was abolished by cytochalasin D but not nocodazole (Figure 5C and 5D). The transport of mitochondria is known to be microtubule-dependent and sensitive to nocodazole [45-47]. Therefore, we further verify that $2 \mathrm{~h}$ treatment with 10 $\mu \mathrm{g} / \mathrm{ml}$ nocodazole was sufficient to inhibit the microtubule-dependent dendritic trafficking by tracking mitochondria movement under the same condition. The result showed that the movement of mitochondria labeled with mito-tracker in living neurons was effectively disrupted by nocodazole (Supplementary information, Movies S1 and S2). These results suggested that the newly inserted GluN2A subunits are trafficked independent of microtubule transport, and thus via the non-canonical pathway.

So far, we have shown that a portion of GluN2A co-localized with the ER chaperone Bip in dendrites, and GluN2A specifically interacted with Bip, which suggested that the GluN2A subunits were reserved in the ER in a Bip-binding form to fulfill the requirement for surface expression during LTP. We further confirmed that the newly inserted GluN2A was transported from the dendritic but not the somatic ER because its rapid insertion was blocked by an actin inhibitor but not a microtubule inhibitor. In summary, these data suggested that GluN2A is transported through the non-canonical trafficking pathway, which bypasses the somatic Golgi and is independent of microtubules.

Interaction of GluN2A with Bip is required for the synaptic increase of the GluN2A-containing NMDARs

To further determine whether Bip is required for the neuronal activity-dependent surface expression of Glu$\mathrm{N} 2 \mathrm{~A}$, we used peptides to interrupt the interaction between GluN2A and Bip. Since it is the GluN2A ATD that contains an ER retention signal and interacts with Bip, we first set out to explore whether the ATD is required for the synaptic insertion of GluN2A. We generated two chimeras with switched ATDs, YFP-GluN2A-ATD ${ }_{2 \mathrm{~B}}$ and 
A

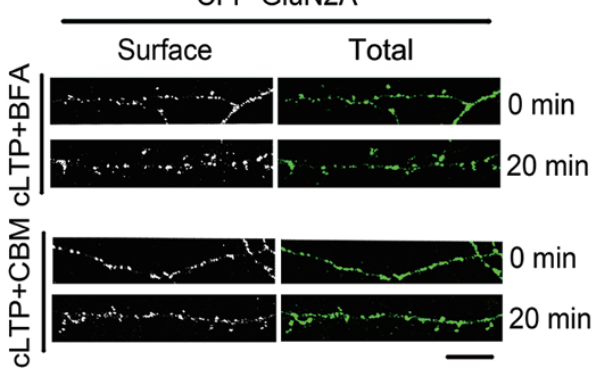

C

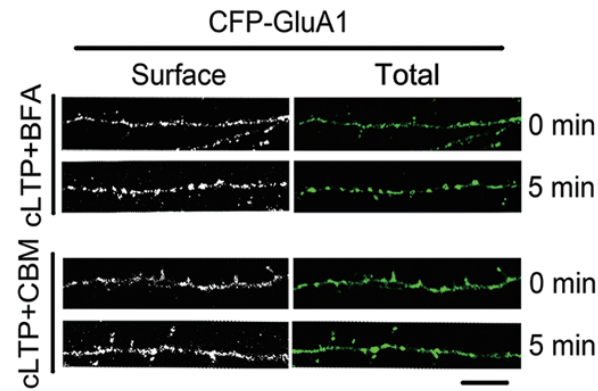

E

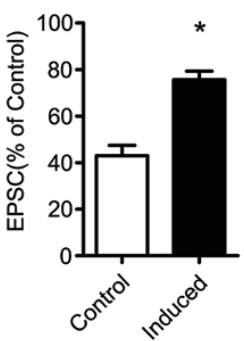

G

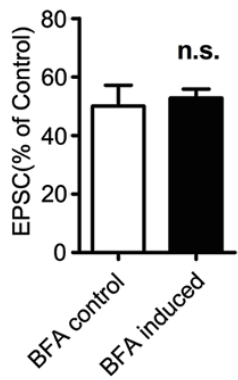

F

Naive

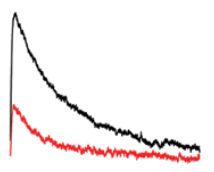

$20 \mathrm{pA}$

$200 \mathrm{~ms}$

$\mathrm{H}$

BFA

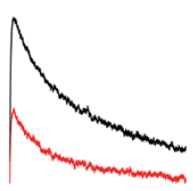

20 pA

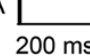

B

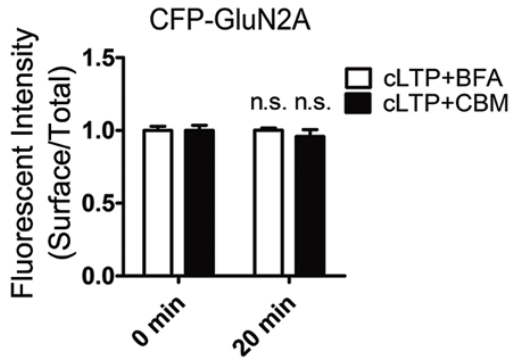

CFP-GluA1

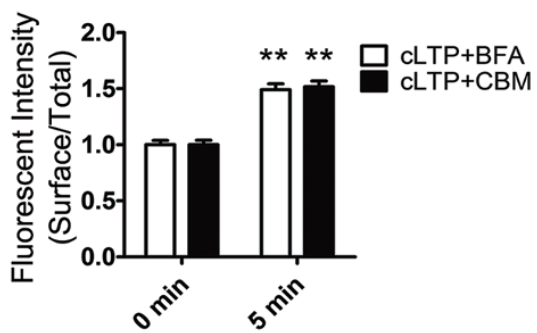

Induced

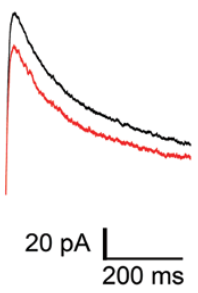

BFA

induced

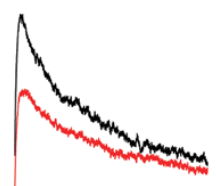

$20 \mathrm{pA}$

$200 \mathrm{~ms}$

Figure 4 The surface increase of GluN2A induced by neuronal activity is abolished by inhibiting ER-to-Golgi trafficking. (A, C) The ER-to-Golgi trafficking inhibitor BFA or CBM was applied to cultured cortical neurons simultaneously with cLTP, and the surface expression of CFP-GluN2A and CFP-GluA1 was assessed by immunostaining. Scale bar $=50 \mu \mathrm{m}$. (B) Quantification of surface expression of CFP-GluN2A after CLTP in the presence of BFA or CBM. After CLTP with BFA: 100.2\% $\pm 1.6 \%, n=$ 33; after CLTP with CBM: $95.8 \% \pm 4.7 \%, n=26$. All data are presented as mean \pm SEM. (D) Quantification of surface expression of CFP-GluA1 after CLTP. Five minutes after CLTP, surface CFP-GluA1 increased in the presence of BFA or CBM. After CLTP with BFA: $149.1 \% \pm 5.3 \%, n=22$; after CLTP with CBM: $151.8 \% \pm 5.2 \%, n=25$. All data are presented as mean \pm SEM. ${ }^{* *} P<0.01$. (E) Quantification of residual NMDA currents of naive group $(43.04 \% \pm 4.36 \%, n=7)$ and induced group $(75.64 \%$ $\pm 3.65 \%, n=6$ ) after application of $3 \mu \mathrm{M}$ ifenprodil. All data are presented as mean \pm SEM. ${ }^{*} P<0.05$. (F) Sample traces of NMDAR EPSCs (black) and residual NMDA current after ifenprodil incubation (red) in control group (naive) and after cLTP induction (induced). (G) Quantification of residual NMDA current in the BFA-only group $(50.05 \% \pm 7.14 \%, n=4)$ and the BFA with induction group $(52.77 \% \pm 3.11 \%, n=6)$ after application of $3 \mu \mathrm{M}$ ifenprodil. All data are presented as mean \pm SEM. $(\mathrm{H})$ Sample traces of NMDAR EPSCs (black) and residual NMDA current after ifenprodil incubation (red), in the presence of BFA (BFA), or with BFA and LTP induction (BFA induced). 
YFP-GluN2B-ATD ${ }_{2 \mathrm{~A}}$ (Figure 6A) and found that the surface expression decreased in both mutants after cLTP induction (Figure 6B and 6C). These data suggested that the GluN2A ATD is necessary for the surface expression of GluN2A-containing NMDARs during neuronal activation. We then attempted to design a peptide to interrupt the interaction between the ATD of GluN2A and the SBD domain of Bip. Since the crystal structure of neither component has been solved, we simulated their structures based on their homologous proteins GluN2B and HSP70 and then the interaction of GluN2A-ATD and Bip-SBD (Figure 7A). Three sequences derived from the ATD of GluN2A at the interface between GluN2A and Bip were used for the synthesis of disrupting peptides. These peptides were fused to the cell membrane trans- duction domain of HIV-1 TAT. The amino-acid sequences for the three peptides were: PEP-1 (YGRKKRRQRRRLKIMQDYDWHV), PEP-2 (YGRKKRRQRRRRSLGLTGYDFF) and PEP-3 (YGRKKRRQRRRELIPKEFPSGLI). Thirty-minute treatment with $10 \mu \mathrm{M}$ of any of the disrupting peptides was enough to dissociate GluN2A-ATD from Bip in transfected HEK293 cells (Figure 7B). Furthermore, exposure to $20 \mu \mathrm{M}$ of any peptide for $30 \mathrm{~min}$ interrupted the interaction between Bip and GluN2A in cultured cortical neurons (Figure 7C-7E and Supplementary information, Figure S3A). However, neither the association between GRP94 and Bip nor that between GluN1 and GluN2A was altered by these peptides (Figure 7C, 7D and Supplementary information, Figure S3A and S3C). The control scrambled
A

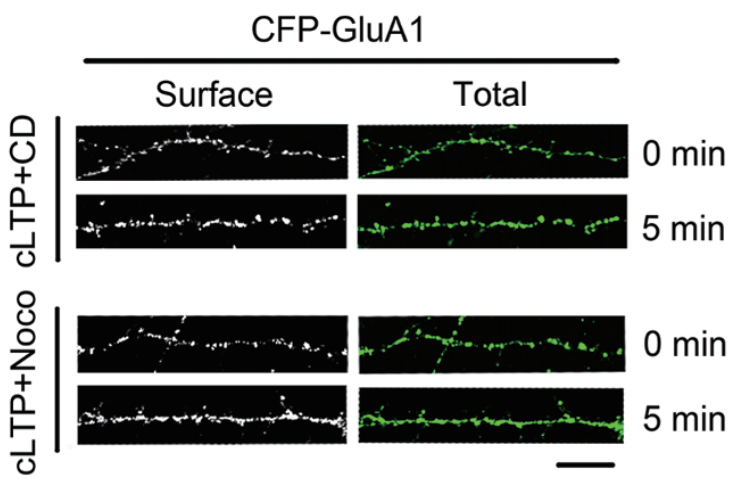

C

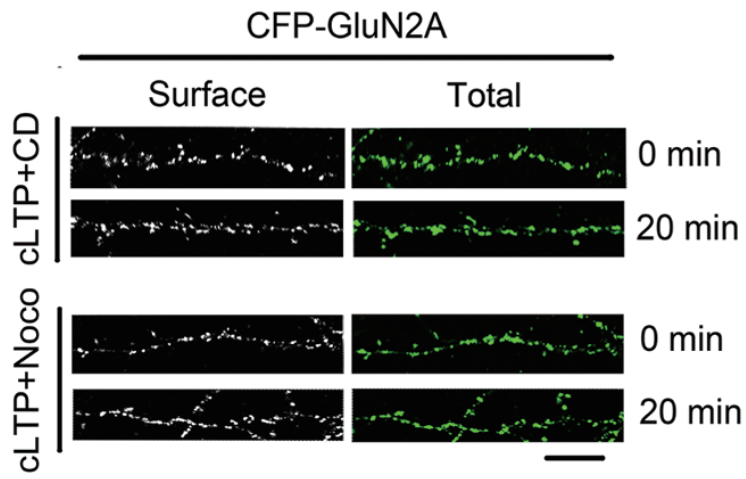

B
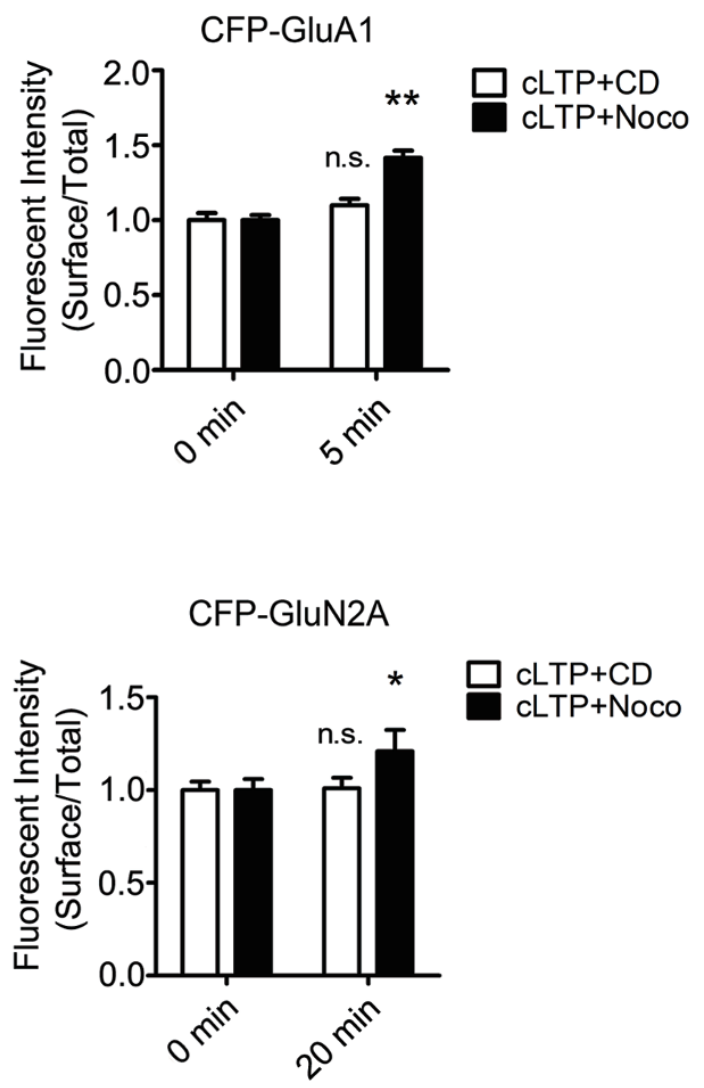

Figure 5 The surface increase of GluN2A-containing NMDARs induced by neuronal activity is abolished by cytochalasin D but not nocodazole. (A, C) Cultured cortical neurons were incubated with the actin inhibitor cytochalasin D or the microtubule inhibitor nocodazole for $2 \mathrm{~h}$, and then subjected to cLTP stimulation. The surface expression of CFP-GluA1 and CFP-GluN2A was assessed by immnunoblotting. Scale bar $=50 \mu \mathrm{m}$. CD, cytochalasin D; Noco, nocodazole. (B) Quantification of CFPGluA1 surface expression. Five minutes after CLTP, surface CFP-GluA1 significantly increased in the presence of Noco, but not changed in the presence of CD. After CLTP with CD: $109.9 \% \pm 4.32 \%, n=22$; after cLTP with Noco: $141.6 \% \pm 4.85 \%$, $n=24$. (D) Quantification of CFP-GluN2A surface expression.CD inhibited the surface increase of CFP-GluN2A, while the increase was not affected by nocodazole. After cLTP with CD: $101 \% \pm 5.7 \%, n=21$; after cLTP with Noco: $120.9 \% \pm 11.5 \%$, $n=24$. All data are presented as mean \pm SEM. ${ }^{*} P<0.05,{ }^{* *} P<0.01$. 


\section{A GluN2A-ATD $28 / G l u N 1 \quad$ GluN2B-ATD $2 A / G l u N 1$}
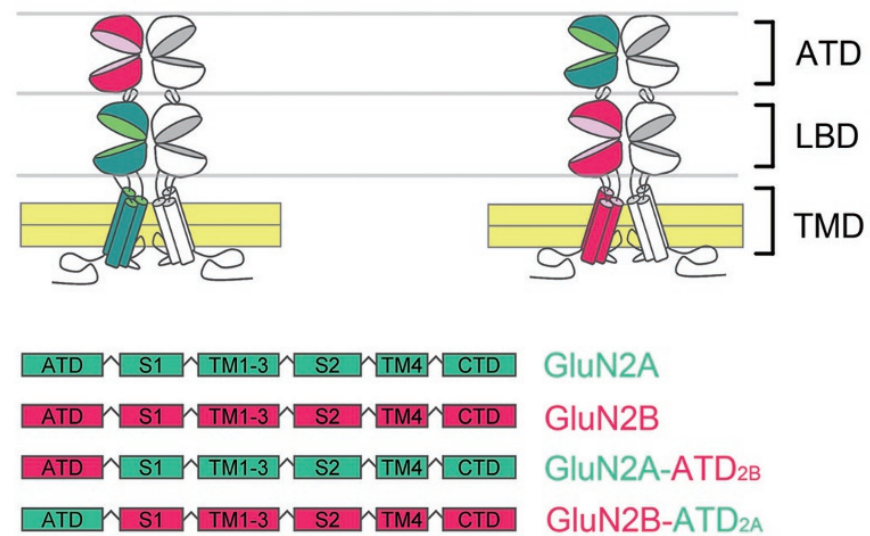

B

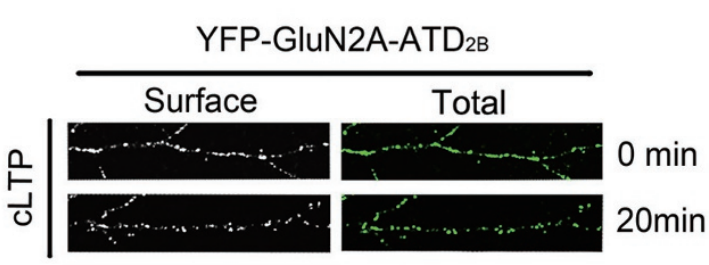

C
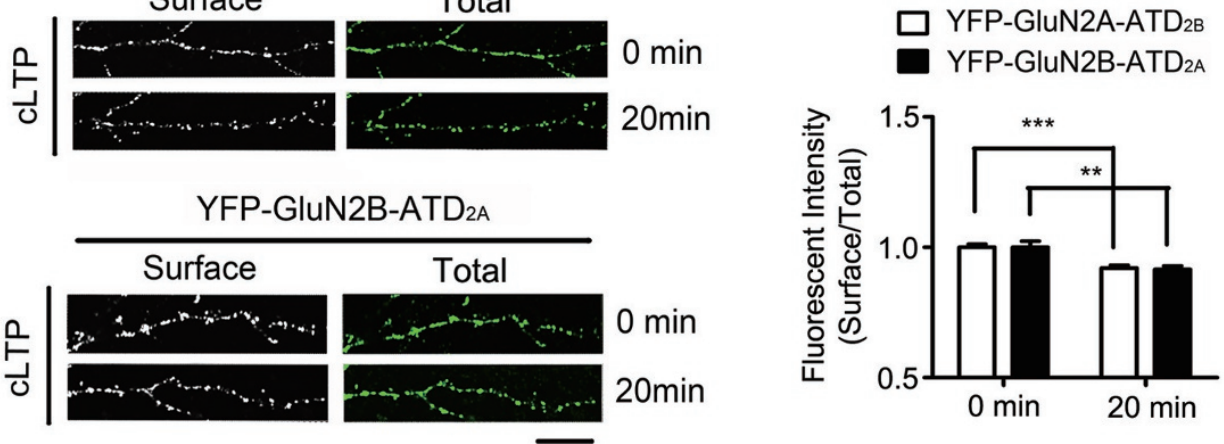

Figure 6 The ATD mediated the surface increase of the GluN2A-containing NMDAR during cLTP. (A) Schematic structures of GluN2A-ATD2B and GluN2B-ATD2A. GluN2A is indicated in green and GluN2B in red; the ATDs of GluN2A and GluN2B were switched with their colors unaltered. (B) Surface expression was changed in YFP-GluN2A-ATD $2 B$ and YFP-GluN2B$\mathrm{ATD}_{2 \mathrm{~A}}$ after cLTP. Scale bar $=50 \mu \mathrm{m}$. (C) Quantification of the surface expression of YFP-GluN2A-ATD ${ }_{2 B}$ and YFP-GluN2B$\mathrm{ATD}_{2 \mathrm{~A}}$ after cLTP, both of which decreased. YFP-GluN2A-ATD $2 \mathrm{~B}: 92 \% \pm 1.1 \%, n=50$; YFP-GluN2B-ATD $2 \mathrm{~A}: 91.5 \% \pm 1.4 \%, n=$ 61. All data are presented as mean \pm SEM. ${ }^{* *} P<0.01,{ }^{* * *} P<0.001$.

peptides had no effects (Figure 7C and Supplementary information, Figure S3B), confirming the specificity of the disrupting peptides. These results also suggested that GluN1 and GluN2A subunits were not spontaneously assembled when GluN2A dissociated from Bip. Then, we assessed whether the disrupting peptides inhibited the neuronal activity-dependent surface expression of GluN2A-containing NMDARs. We first incubated cultured cortical neurons with $20 \mu \mathrm{M}$ of each of the disrupting peptides PEP-1, PEP-2, and PEP-3 for $1 \mathrm{~h}$, and then analyzed the surface expression of CFP-GluN2A before and after cLTP induction. We found that the surface increase of CFP-GluN2A was abolished by any of the disrupting peptides but not the scrambled peptides (Figure 8A and Supplementary information, Figure S4). We also as- sessed whether the membrane insertion of CFP-GluA1 was affected in a nonspecific manner. When we incubated CFP-GluA1-transfected cortical neurons with a combination of $60 \mu \mathrm{M}$ of PEP-1, PEP-2 and PEP-3 for $1 \mathrm{~h}$ and then applied cLTP, the surface CFP-GluA1 still increased (Figure 8B). We also analyzed whether the peptides inhibited the LTP-induced switch of the GluN2Bto GluN2A-containing NMDARs in hippocampal slices as described in the above experiments, and found that intracellular infusion of PEP-2 $(2.3 \mu \mathrm{M})$ but not SCR-2 effectively blocked an increase in the GluN2A-containing NMDAR currents (Figure 8C and 8D). These results showed that after the Bip and GluN2A interaction was disrupted by PEP-2 derived from the GluN2A ATD, Glu$\mathrm{N} 2 \mathrm{~A}$ neither responded to neuronal activity nor inserted 
A
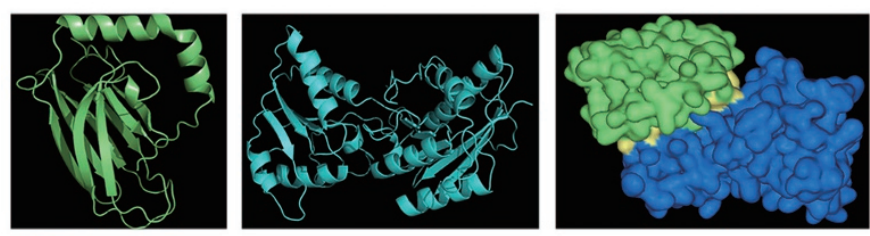

SBD of Bip

ATD of GluN2A
$B$

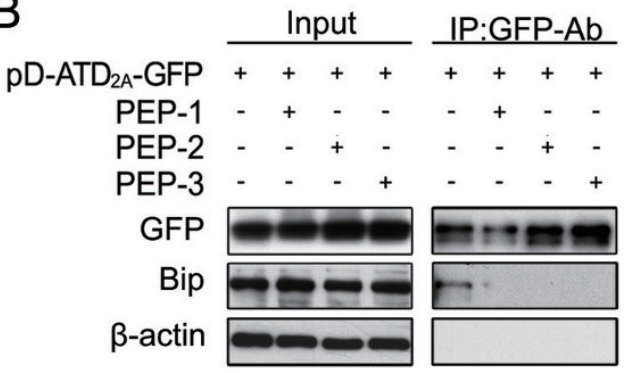

$\mathrm{E}$

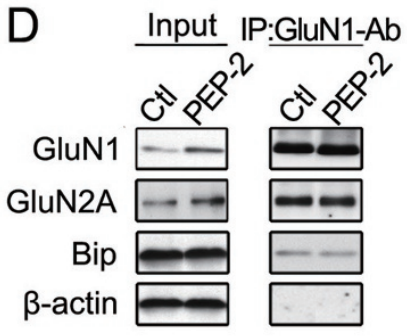

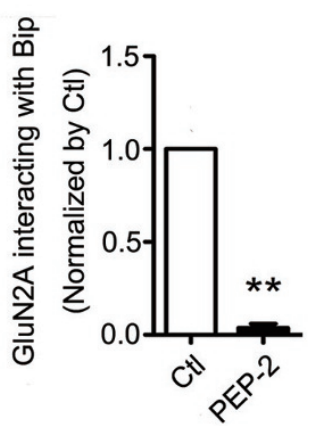

Figure 7 Peptides derived from the ATD of GluN2A interrupt the interaction between GluN2A and Bip. (A) Modified structure of the SBD domain of Bip (left, green) and the ATD of GluN2A (right, blue). Simulated structure of the binding complex of BipSBD (green), GluN2A-ATD (blue) and their interaction interface (yellow). (B) HEK293 cells transfected with pD-ATD2A-GFP were exposed to $20 \mu \mathrm{M}$ of each peptide (PEP-1, PEP-2 and PEP-3) for $0.5 \mathrm{~h}$, then cell extracts were co-immunoprecipitated by rabbit anti-GFP antibody. Actin was used as negative control $(n=4)$. (C) Cultured cortical neurons were exposed to 20 $\mu \mathrm{m}$ of the disrupting peptide (PEP-2) or scrambled peptide (SCR-2) for $1 \mathrm{~h}$, then cell extracts were co-immunoprecipitated by rabbit anti-Bip antibody. GRP94 was used as positive control $(n=4)$. (D) Cultured cortical neurons were exposed to $20 \mu \mathrm{m}$ PEP-2 for $1 \mathrm{~h}$, then cell extracts were co-immunoprecipitated by rabbit anti-GluN1 antibody $(n=4)$. (E) Quantification of the effects of PEP-2 on disrupting the interaction between GluN2A and Bip. Level of GluN2A-Bip binding in the presence of PEP2: $3.66 \% \pm 4.5 \%, n=4$. All data are presented as mean \pm SEM. ${ }^{* *} P<0.01$.

into the synaptic membrane.

Interfering with the interaction between Bip and GluN2A in vivo interrupts the acquisition of fear memory

It is known that the subunit composition of the NMDAR changes during neuronal activation $[42,48]$, but the physiological significance of this change is not clear. Therefore, we investigated whether the peptide PEP-2 also functioned in vivo as it did in vitro. First, mice were given an intraperitoneal injection of $10 \mu \mathrm{m} / \mathrm{kg}$ PEP-2. One hour later the hippocampus was dissected out and the Bip-bound GluN2A was co-immunoprecipitated and measured. The results showed that the interaction between GluN2A and Bip was effectively disrupted by PEP-2, but not SCR-2 (Figure $8 \mathrm{E}$ and $8 \mathrm{~F}$ ). Next, the fEPSP before and after LTP-inducing stimulation was recorded in the SC-CA1 synapse of mouse hippocampal slices harvested $1 \mathrm{~h}$ after injection of SCR-2 or PEP-2. The results showed that injection of PEP-2, but not SCR-
2 significantly impaired the expression of LTP (Figure $8 \mathrm{G}$ and $8 \mathrm{H})$. We tested whether the injection of PEP-2 affected fear conditioning. Mice were injected with PEP$21 \mathrm{~h}$ before training, three conditioned stimuli (CS) and an unconditioned stimulus (US) paired trials were conducted as training (Figure 8I). Twenty-four hours after training, the mice were placed in the training chamber and the freezing behavior of each mouse was assessed in the presence of the CS or US. The results showed that the formation of contextual fear memory was impaired by PEP-2 but not SCR-2 (Figure 8J). Two days after the contextual fear test, the cued fear conditioning was again evaluated. Consistently, the cued fear conditioning was impaired by PEP-2, but not SCR-2 (Figure 8K). Since the peptide was injected before training, these data suggested that the acquisition of fear conditioning was impaired by the peptide, which indicates that the association between GluN2A and Bip is a critical factor in memory formation. 
A

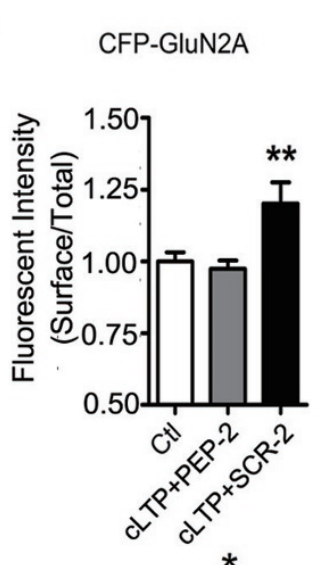

D

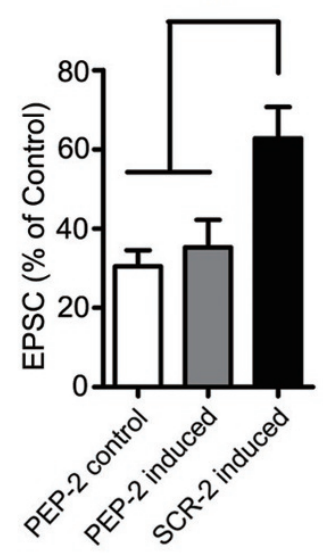

G

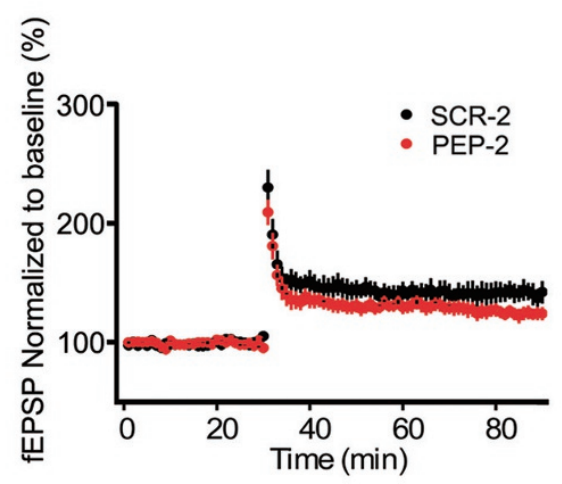

H

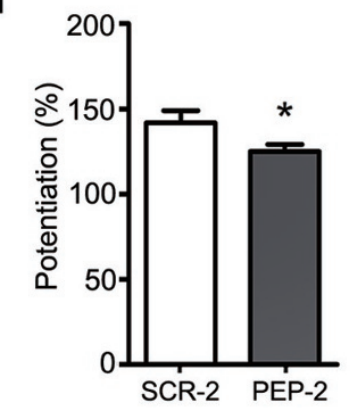

E

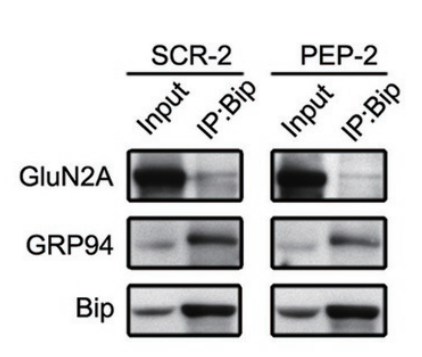

$\mathrm{F}$
C

PEP-2 PEP-2 SCR-2

control induced induced
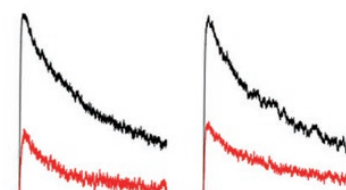

$20 \mathrm{pA} L 200 \mathrm{~ms}$

$20 \mathrm{pA} \bigsqcup_{200 \mathrm{~ms}}$

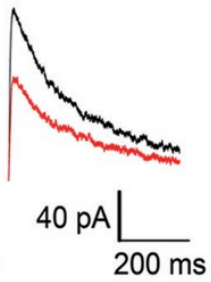

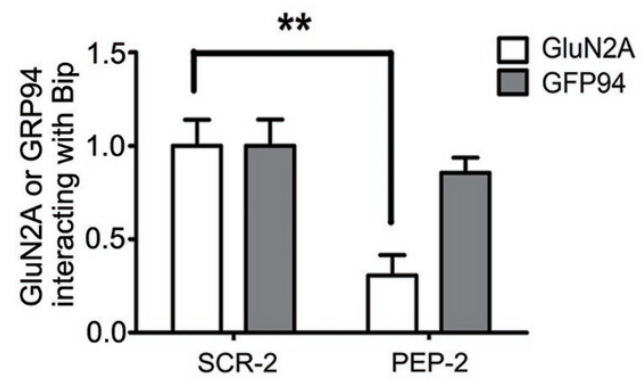

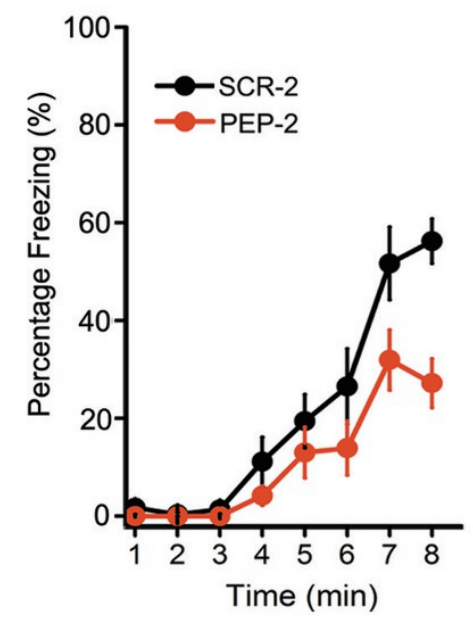

J

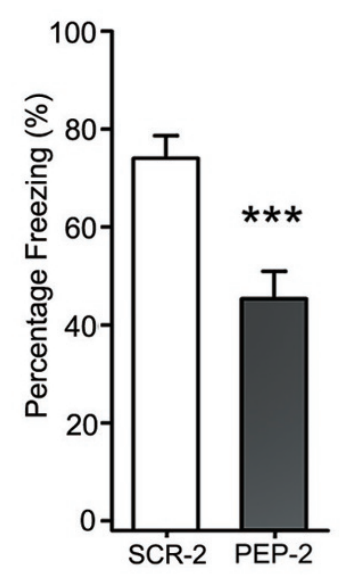

K

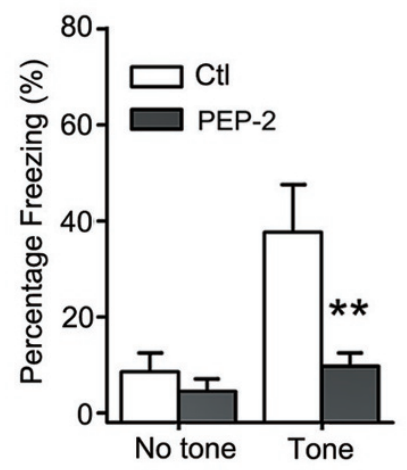

www.cell-research.com | Cell Research 
Figure 8 Effects of disrupting peptides in vitro and in vivo. (A) Quantification of the effects of a disrupting peptide and the scrambled peptide on the surface expression of the GluN2A-containing NMDARs induced by neuronal activity. cLTP with PEP-2: $98.3 \% \pm 2.9 \%, n=31$; cLTP with SCR-2: $115.4 \% \pm 5.0 \%, n=21$. All data are presented as mean \pm SEM. ${ }^{*} P<0.05$; ${ }^{* *} P<0.01$. (B) Quantification of the effects of a mixture of all three peptides (PEP-t) on the surface expression of GluA1-containing AMAPRs induced by neuronal activity. cLTP with PEP-t: $155 \% \pm 9.3 \%, n=17$. ${ }^{* *} P<0.01$. (C) Sample traces of NMDAR EPSCs (black) and residual current after ifenprodil incubation (red) in control group (peptide control), cLTP induction with PEP-2 (peptide induced) and CLTP induction with SCR-2 (scrambled peptide induced). (D) Quantification of residual NMDAR currents in the peptide control group $(30.46 \% \pm 4.10 \%, n=6)$, peptide treatment with induction $(35.25 \% \pm 6.98 \%$, $n=7)$, and scrambled peptide treatment with induction $(62.80 \% \pm 7.91 \%, n=6)$, ${ }^{\star} P<0.05$. (E) The interaction between Bip and GluN2A was evaluated by co-IP with antibody against Bip $1 \mathrm{~h}$ after injection of the interrupting peptide PEP-2 or the scrambled peptide SCR-2, and the interaction between GRP94 and Bip was taken as control. (F) Quantification of the interaction between Bip and GluN2A. After injection of the interrupting peptide, the interaction between GluN2A and Bip declined to $30.7 \% \pm 14 \%(n=5)$ compared to the group with scrambled peptide injection. ${ }^{* *} P<0.01$. (G) Trace of fEPSP in hippocampal slices before and after LTP induction recorded in mice after injection of SCR-2 or PEP-2. (H) Potentiation of the fEPSP slope (normalized to baseline) at $60 \mathrm{~min}$ after post-tetanic induction. SCR-2: $141.77 \% \pm 7.17 \%, n=8 ; \mathrm{PEP}-2: 129 \% \pm 4.21 \%$, $n=9$. ${ }^{*} P<0.05$. (I) Fear conditioning in mice injected with interfering peptide (PEP-2) was significantly impaired compared to control (injected with scrambled peptide). Control, $n=9$; Peptide, $n=10$. (J) Twenty-four hours after training, SCR-2-treated mice showed a longer freezing time than PEP-2-treated mice in the contextual fear conditioning test. SCR-2, 74.03\% $\pm 4.43 \%$; PEP-2, 45.38\% $\pm 5.44 \%$. ${ }^{* * *} P<0.001$. (K) Forty-eight hours after contextual fear conditioning, PEP-2-treated mice had less freezing in response to the auditory cue. No Tone (cued fear conditioning test without conditioned stimuli): Ctl, $8.57 \% \pm 3.68 \%$; PEP-2, 4.50\% $\pm 2.43 \%$; Tone (cued fear conditioning with conditioned stimuli): Ctl, $37.67 \% \pm 9.86 \%$; PEP-2, 9.77\% $\pm 2.58 \%$. ${ }^{* *} P<0.01$.

\section{Discussion}

Non-canonical trafficking of GluN2A-containing NMDARs during neuronal activation

Synaptic NMDAR subunit composition is dynamically regulated during development and neuronal activation, and correspondingly alters synaptic plasticity. Different trafficking pathways and dynamic regulation of the GluN2A- and GluN2B-containing NMDARs provide a critical mechanism to ensure their appropriate synaptic function in the adult forebrain $[6,7,10,12]$. The surface expression of GluN2B-containing NMDARs can be changed by inhibiting or promoting its recycling [49-51], or by lateral diffusion [14], while GluN2A-containing NMDARs are primarily located on synapses and much less mobile [16, 52], therefore the latter seems to be regulated in ways other than recycling or lateral diffusion. It has recently been suggested that both GluN2A and GluN2B can be transported via a non-canonical pathway in which they are transported along dendrites within ER $[23,24]$, and it has been demonstrated that during cLTP the dendritic synthesis of GluN2A is initiated $[25,26]$. This evidence suggested that the dynamic expression of the GluN2A-containing NMDAR during neuronal activation may depend on non-canonical trafficking. We have provided new evidence for this by showing that the synaptic insertion of GluN2A-containing NMDARs induced by the cLTP protocol depends on their delivery from the ER, and further we demonstrated that microtubules are not required for the transport of GluN2A during this process, which indicates that the GluN2A is indeed delivered directly from the dendrite but not the soma. All together, these results showed that the transport of GluN2A was through the non-canonical trafficking pathway. Previous work has shown that dendrites contain key elements for the local synthesis of membrane proteins, such as mRNA, dendritic ER and Golgi outpost [53-55]; however, more interesting is the neuronal activity-dependent regulation of non-canonical trafficking and its importance in neuronal plasticity. Here, we found that interfering the non-canonical trafficking of GluN2A disrupted its synaptic insertion, and the LTP and fear conditioning of mice were also affected; all together these results suggest an important role of non-canonical trafficking in neuronal plasticity.

\section{A reserve pool of GluN2A in dendritic ER}

In this study, we uncovered a novel mechanism of non-canonical trafficking of GluN2A-containing NMDARs to synapses. We revealed that the ER chaperone Bip reserves GluN2A subunits in the dendritic ER and mediates the assembly and delivery of GluN2A-containing NMDARs at the onset of LTP induction. We also found that Bip selectively interacted with GluN2A and they were partly co-localized in dendrites, indicating the storage of GluN2A subunits inside the dendritic ER. Besides, we found that the co-localization between GluN2A and Bip significantly decreased after cLTP induction, accompanied by an increase of co-localization between GluN2A and PSD95. We also found a decrease of inter- 
action between Bip and GluN2A that was accompanied by a simultaneous increase in the interaction between GluN2A and GluN1. These data suggested a transfer of GluN2A from a Bip-bound form inside the dendritic ER to the fully assembled functional receptor expressed in synapses, and this transfer was regulated by neuronal activity. In addition, we found that this mechanism was specific for GluN2A, since GluN2B barely interacted with Bip, and mutant GluN2A with its ATD replaced by that of GluN2B was not delivered to the surface in response to neuronal activity. Thus, the Bip-bound form of GluN2A in the dendritic ER actually serves as a reserve pool of GluN2A subunits for assembly and delivery of its functional receptors. Clearly, this is a different strategy from that of the AMPAR, where the newly inserted synaptic AMPAR in response to neuronal activity mainly comes from recycling vesicles within the dendritic spine; i.e., it is a reserve pool of functional receptors [56]. Similarly, this endosome-dependent recycling is also the mechanism by which the synaptic expression of GluN2B-containing NMDARs is regulated. Such a particular arrangement of GluN2A storage may reflect that the synaptic incorporation of the GluN2A-containing NMDAR is more strictly regulated and also time-coordinated.

Bip is a well-studied multi-functional chaperone protein in the ER [57]. The binding of Bip with its substrates is an ATP-dependent process, and the binding efficacy can be regulated, which makes it a good candidate for regulating multi-subunit protein assembly [58]. For example, Bip binds more tightly to the heavy chain of the class I major histocompatibility complex than to its light chain [59]. The dissociation kinetics of Bip and the heavy chain is regulated by ERdj3, which is a co-chaperone of Bip $[58,59]$. Similarly, we found that Bip specifically interacted with GluN2A but not GluN1 or GluN2B, which depends on the ATD of GluN2A. Since the ATD of GluN2A is localized inside the ER lumen when GluN2A is newly synthesized, the neuronal activity signal needs to be transduced from the cytoplasm to the ER lumen in order for Bip to modulate the assembly of NMDAR subunits through the ATD of GluN2A. There may be two avenues for this signal transduction: through trans-ERmembrane proteins, or through $\mathrm{Ca}^{2+}$-sensitive chaperone proteins resident in the ER lumen, since the $\mathrm{Ca}^{2+}$ concentration inside the ER is markedly reduced during LTP. Bip seems to fulfill both requirements. First, it has several co-chaperones that are transmembrane proteins on the ER. For example, ERdj1 is one of the Bip co-chaperones whose cytosolic tail is phosphorylated in vitro by CK2 [59]. Second, the ATPase activity of Bip is altered by $\mathrm{Ca}^{2+}[60]$, thus the decrease of $\mathrm{Ca}^{2+}$ concentration in the
ER lumen upon neuronal activation may lead to direct dissociation of Bip from GluN2A. Recently it has been found that activation of the sigma- 1 receptor, which is also an ER chaperone, leads to the surface expression of NMDARs [61]. Interestingly, sigma-1 binds directly with Bip and the interaction of these two chaperones is regulated by the $\mathrm{Ca}^{2+}$ level inside the ER $[62,63]$. Since the $\mathrm{Ca}^{2+}$ concentration in the ER is reduced during LTP, it is possible that the sigma-1 receptor and Bip coordinately regulate NMDAR expression. However, there may be other means by which the signal is transduced into the ER, and future studies are needed to elucidate the mechanism for the coupling of neuronal plasticity with the dissociation of GluN2A from Bip.

\section{Role of the synaptic expression of GluN2A-containing NMDARs in fear conditioning}

The surface expression of NMDARs, as well as their subunit composition is dynamically regulated by neuronal activity in the hippocampus [64-66], but the role of the newly inserted NMDARs in hippocampal LTP or animal behavior is unclear. The existing evidence suggests that the NMDAR is upregulated to re-establish the baseline AMPAR/NMDAR ratio [67], or to modify the induction threshold for AMPAR-mediated plasticity which is known as metaplasticity $[2,68]$. In this study, we showed that the maintenance of hippocampal LTP was impaired by in vivo application of a peptide that selectively inhibits the forward trafficking of GluN2A-containing NMDARs. This implied that the newly expressed synaptic GluN2A-containing NMDARs are required in hippocampal LTP. More importantly, we provided evidence for a direct link between the molecular modification of LTP and animal behavior, the establishment of fear conditioning. However, further study is needed to assess the possible relationship between impaired hippocampal LTP and the failure to establish fear conditioning.

In parallel with investigations into the possibility of a subunit-selective contribution to plasticity, subunit-specific procedures were also used to analyze whether GluN2A and GluN2B are important for animal behaviors. For instance, GluN2A or GluN2B knockout has been used to study the consequences of the developmental switch in the composition of NMDARs from GluN2B-dominant to GluN2A-dominant [69] and to study the distinct functions of the GluN2A- or GluN2B-containing NMDARs in controlling behavior $[69,70]$. Clearly, the weakness of using the knockout approach is that the long-term depletion of a particular subunit may lead to other changes in the entire neuronal network and may also induce compensatory processes. Furthermore, when using knockouts, it is difficult to 
study changes in NMDAR composition at short-time scales, especially in the mature brain where both the GluN2A- and GluN2B-containing NMDARs are already extensively expressed. However, we found that peptide application changed neither the total nor the surface expression of GluN2A significantly while it effectively interrupted the Bip and GluN2A interaction and inhibited the synaptic insertion of GluN2A-containing NMDARs in response to synaptic activity. These advantages make the peptide ideal for in vivo study of the role of the synaptic GluN2A-containing NMDARs in memory formation in mature rodents. In our study, we tested the effect of intraperitoneal injection of the peptide on fear conditioning and found that the acquisition of fear memory was impaired. Previous studies have demonstrated that blockade of NMDARs with the non-selective antagonist APV [71] or selective blockade of the GluN2B-containing NMDAR $[72,73]$ impairs the acquisition of fear memory. However, all the antagonists used affect either synaptic plasticity or basic synaptic transmission, since both are themselves NMDAR-mediated processes. In this study, the peptide we generated only inhibited the neuronal activity-induced increase of the GluN2A-containing NMDARs but not the basal levels, in other words the basic synaptic transmission mediated by NMDARs was not affected. Thus, our results provide novel evidence that Bip-mediated and neuronal activity-dependent synaptic insertion of GluN2A-containing NMDARs is required for fear memory formation. Furthermore, this peptide could be used in future for in vivo studies on the function of synaptic increases of the GluN2A-containing NMDARs in other forms of learning and memory.

\section{Materials and Methods}

\section{Plasmid construction}

The plasmids CFP-GluN2A, CFP-GluN2B, YFP-GluN2B-AT$\mathrm{D}_{2 \mathrm{~A}}$, YFP-GluN2A-ATD $2 \mathrm{~B}, \mathrm{pD}-\mathrm{ATD}_{2 \mathrm{~A}}-\mathrm{GFP}, \mathrm{pD}-\mathrm{ATD}_{2 \mathrm{~B}}-\mathrm{GFP}$ and pD-GFP had all been constructed in our previous studies [74]. In brief, CFP-GluN2A and CFP-GluN2B were made by inserting CFP between the signal peptide and the ATD of GluN2A and GluN2B. For YFP-GluN2B-ATD ${ }_{2 \mathrm{~A}}$ and YFP-GluN2A-ATD ${ }_{2 \mathrm{~B}}$, the ATDs were first deleted from GluN2A and GluN2B by conventional DNA mutagenesis, then the ATD of GluN2A was inserted into the ATD-deleted GluN2B mutant and vice versa; the expression vector of $\mathrm{pD}-\mathrm{ATD}_{2 \mathrm{~A}}-\mathrm{GFP}, \mathrm{pD}-\mathrm{ATD}_{2 \mathrm{~B}}$-GFP and $\mathrm{pD}$-GFP was $\mathrm{p}$-Display in which multiple cloning sites are located at the $\mathrm{N}$-terminal side of its encoded transmembrane domain of platelet-derived growth factor receptor (PDGFR). Thus, $\mathrm{ATD}_{2 \mathrm{~A}}-\mathrm{GFP}$ and $\mathrm{ATD}_{2 \mathrm{~B}}$-GFP were derived by PCR from GFP-GluN2A and GFP-GluN2B which were constructed in previous studies [75], and then inserted between the XmaI and SacII sites of p-Display to make a chimera with AT$\mathrm{D}_{2 \mathrm{~A}}$-GFP or $\mathrm{ATD}_{2 \mathrm{~B}}-\mathrm{GFP}$ attached to the transmembrane domain of PDGFR at the C-terminus.

\section{Cortical neuron culture and transfection}

Cortical neuron cultures were prepared from E18 rats following the protocol described previously [76]. Neurons were transfected at DIV 10 using Lipofectamine LTX with PLUS (Invitrogen). Plasmids were first mixed with PLUS reagent and then mixed with Lipofectamine LTX and incubated for $30 \mathrm{~min}$ at room temperature. The final mixture was added to dishes containing neurons and incubated for $3 \mathrm{~h}$. The culture medium collected before transfection was replaced. $2 \mu \mathrm{g}$ of plasmid was used in 35-mm dishes according to the manufacturer's protocol. After 4 days, cultured neurons were used for experiments.

\section{cLTP induction}

The cLTP protocol used was described previously [30]. Neurons were rinsed twice in extracellular solution (ECS; in mM: 140 $\mathrm{NaCl}, 1.3 \mathrm{CaCl}_{2}, 5 \mathrm{KCl}, 35$ HEPES, 33 glucose, $\mathrm{pH}$ 7.4), incubated in Buffer A (in $\mu \mathrm{M}$ : 0.5 TTX, 1 strychnine, 20 bicuculline in ECS, pH 7.4) for $5 \mathrm{~min}$, and in Buffer B $(200 \mu \mathrm{M}$ glycine in Buffer A) for another 5 min. After a 5 -min rinse in ECS, neurons were incubated in Buffer A for another 5 or $20 \mathrm{~min}$. In the control group, Buffer B was replaced by ECS. The neurons were then used for experiments.

\section{Expression analysis by biotinylation and surface staining}

Biotinylation Hippocampal cultures were rinsed twice with icecold PBS (in mM: $137 \mathrm{NaCl}, 2.7 \mathrm{KCl}, 10 \mathrm{Na}_{2} \mathrm{HPO}_{4}, 2 \mathrm{KH}_{2} \mathrm{PO}_{4}$, $\mathrm{pH}$ 7.4) with $1 \mathrm{mM} \mathrm{MgCl}_{2}$ and $0.5 \mathrm{mM} \mathrm{CaCl}_{2}$, then incubated with PBS (with $1 \mathrm{mg} / \mathrm{ml}$ sulfo-NHS-LC-biotin; Thermo Fisher Scientific) for $30 \mathrm{~min}$ at $4{ }^{\circ} \mathrm{C}$. PBS with $100 \mu \mathrm{M}$ glycine was added to quench the biotin binding for $15 \mathrm{~min}$ at $4{ }^{\circ} \mathrm{C}$. Cells were lysed in ice-cold RIPA buffer $(0.1 \%$ SDS, $0.5 \%$ sodium deoxycholate, $1 \%$ Triton X-100) after rinsing twice with PBS, and incubated at $4{ }^{\circ} \mathrm{C}$ for $30 \mathrm{~min}$. Cells were concentrated at $12000 \times \mathrm{g}$ for $10 \mathrm{~min}$, and incubated with NeutrAvidin beads (Thermo Fisher Scientific) for $2 \mathrm{~h}$ at $4{ }^{\circ} \mathrm{C}$. The beads were then washed three times with RIPA buffer, and proteins were extracted with $2 \times$ sample buffer and boiled at $100{ }^{\circ} \mathrm{C}$ for $10 \mathrm{~min}$ before use for western blot.

Staining Cortical neurons plated on slides were rinsed three times in ECS, fixed in 4\% paraformaldehyde for $5 \mathrm{~min}$, and blocked with $5 \%$ BSA in ECS for $10 \mathrm{~min}$. Then the neurons were incubated in home-made rabbit anti-GFP primary antibody (1:500) for $15 \mathrm{~min}$, rinsed three times in ECS, and incubated with Alexa-546-conjugated secondary antibody (1:1 000) for another $15 \mathrm{~min}$. After rinsing in ECS for $10 \mathrm{~min}$, neurons were blocked and permeabilized with $0.1 \%$ Triton X-100 in PBS containing 5\% BSA. They were then incubated in primary and secondary antibodies for $1 \mathrm{~h}$ each, with three rinses before and after incubation with secondary antibody. The neurons were examined under a $60 \times, 1.4$ numerical aperture oil-immersion objective on an Olympus confocal microscope equipped with FV1000 software. Surface and intracellular staining were analyzed using Metamorph 5.0 software, and the ratio of surface/intracellular signals after stimulation was normalized to the ratio in transfected neurons before stimulation.

For data acquisition from STORM, we used a method previously described [28]. DIV14 hippocampal neurons on coverslips were fixed in $4 \%$ paraformaldehyde for $15 \mathrm{~min}$. After fixation, cells were permeabilized and blocked with 5\% BSA in PBS containing $0.2 \%$ Triton X-100 for 40 min. Primary and secondary antibodies 
were diluted in 5\% BSA and applied at room temperature. For immunostaining, coverslips were inverted on a drop $(60 \mu 1)$ of primary antibody solution (PSD95, 1:60; GluN2A, 1:20; Bip, 1:60) on parafilm for $1 \mathrm{~h}$, then washed three times with PBS for 10 min per wash in a 24-well plate and inverted again on a drop of secondary antibody solution (donkey anti-mouse Alexa-405/Alexa-647, 1:50; donkey anti-rabbit Cy3/Alexa-67, 1:50) for 1h. After washing four times in PBS, the coverslips were post-fixed with $3 \%$ paraformaldehyde $+0.1 \%$ glutaraldehyde in PBS for $20 \mathrm{~min}$ and stored in PBS for immediate STORM microscopy.

\section{Extraction of Triton $X$-insoluble PSD fraction}

After washing twice with ECS, the cells were homogenized in buffer A (320 mM sucrose, $10 \mathrm{mM}$ HEPES, $\mathrm{pH}$ 7.4) and centrifuged at $12000 \times \mathrm{g}$ for $20 \mathrm{~min}$, the supernatant was removed, and the pellet was collected as a crude membrane fraction. The pellet was then dissolved in buffer B (4 mM HEPES, 1 mM EDTA, $\mathrm{pH}$ 7.4) and centrifuged at $12000 \times g$ for another $20 \mathrm{~min}$, and this pellet was further dissolved in buffer C (20 mM HEPES, $100 \mathrm{mM}$ $\mathrm{NaCl}, 0.5 \%$ Triton $\mathrm{X}-100, \mathrm{pH} 7.2)$. After incubation for $15 \mathrm{~min}$ at $4{ }^{\circ} \mathrm{C}$, the homogenate was centrifuged at $12000 \times \mathrm{g}$ for $20 \mathrm{~min}$, and this pellet was the Triton X-100-insoluble PSD fraction. This was dissolved in buffer D (20 mM HEPES, $0.15 \mathrm{mM} \mathrm{NaCl}, 1 \%$ Triton X-100, 1\% deoxycholic acid, 1\% SDS, 1 mM DTT, pH 7.5) and incubated at $4{ }^{\circ} \mathrm{C}$ for $1 \mathrm{~h}$. After centrifugation at $10000 \times \mathrm{g}$ for $15 \mathrm{~min}, 4 \times$ sample buffer was added to the supernatant and the mixture was boiled at $100{ }^{\circ} \mathrm{C}$ for $10 \mathrm{~min}$ before use for western blot.

\section{Glutathione S-transferase (GST) pull-down assay}

After washing glutathione sepharose beads twice with PBS and once with binding buffer $(50 \mathrm{mM}$ Tris $\cdot \mathrm{HCl}, 0.1 \%$ Triton $\mathrm{X}-100, \mathrm{pH} 7.4), 10 \mu \mathrm{g}$ GST or $10 \mu \mathrm{g}$ GST-SBD $_{\text {Bip }}$ was incubated with the beads in $500 \mu \mathrm{l}$ binding buffer at $4{ }^{\circ} \mathrm{C}$ for $2 \mathrm{~h}$. The beads were washed three times with binding buffer, the supernatant was removed, and $500 \mu 1$ rat brain tissue (extracted by a non-denaturing method) was added and incubated overnight. The beads were washed three times with binding buffer, $2 \times$ sample buffer was added, and the mixture was boiled at $100{ }^{\circ} \mathrm{C}$ for $10 \mathrm{~min}$ before use for western blot.

\section{Electrophysiological recording in hippocampal slices}

7-11-day-old Sprague-Dawley rats were anesthetized with ether and decapitated. Transverse hippocampal slices $(350 \mu \mathrm{m}$ thick) were cut in ice-cold artificial cerebrospinal fluid (ACSF) containing (in mM): $119 \mathrm{NaCl}, 2.5 \mathrm{KCl}, 2.5 \mathrm{CaCl}_{2}, 1.3 \mathrm{MgSO}_{4}, 1.0$ $\mathrm{NaH}_{2} \mathrm{PO}_{4}, 26.2 \mathrm{NaHCO}_{3}$ and 11 glucose, equilibrated with $95 \% \mathrm{O}_{2}$ and $5 \% \mathrm{CO}_{2}$. The slices were then allowed to recover for $30 \mathrm{~min}$ in ACSF at $37{ }^{\circ} \mathrm{C}$, followed by at least $30 \mathrm{~min}$ recover at room temperature before recording. Whole-cell patch-clamp recordings were made from visually identified CA1 pyramidal neurons in the presence of $100 \mu \mathrm{M}$ picrotoxin at $30^{\circ} \mathrm{C}$. The pipette solution contained (in mM): $115 \mathrm{CsMeSO}_{4}, 20 \mathrm{CsCl}_{2}, 10 \mathrm{HEPES}, 2.5 \mathrm{MgCl}_{2}, 4$ NaATP, 0.4 NaGTP, 10 NaPhosphocreatine and 0.6 EGTA (pH 7.2). Excitatory postsynaptic currents (EPSCs) were evoked by stimulation of Schaffer collateral/commissural axons at $0.1 \mathrm{~Hz}$ using concentric bipolar electrodes placed in the stratum radiatum of CA1 in the presence of $100 \mu \mathrm{M}$ picrotoxin. Recordings were made using a MultiClamp 700B patch-clamp amplifier (Axon Instruments,
Foster City, CA, USA); signals were filtered at $3 \mathrm{kHz}$, digitized at $10 \mathrm{kHz}$, and analyzed using pClamp 10.2 (Axon Instruments). Recordings in which the access resistances changed by $>10 \%$ were excluded from analysis. As previously described [41], switching of NMDAR subunit composition was induced by a pairing protocol that was used to induce LTP, composed of a depolarization to $0 \mathrm{mV}$ in the postsynaptic cell and $2 \mathrm{~Hz} / 180$ pulse stimulation at Schaffer collateral/commissural axons. Induction success was determined by an AMPAR-mediated current increase after delivery of the protocol; NMDAR-mediated EPSCs were then recorded in the additional presence of $10 \mu \mathrm{M} C N Q X$ and voltage-clamped at $+40 \mathrm{mV}$. To identify the subunit composition of the NMDARs, 3 $\mu \mathrm{M}$ ifenprodil (a GluN2B blocker) was applied for 25-30 min until no further decrease of the NMDA EPSC was observed. BFA (20 $\mu \mathrm{M})$ and peptides $(2.3 \mu \mathrm{M})$ were applied in the internal solution throughout the recording procedure.

\section{Electrophysiological recording after in vivo injection of peptide}

Transverse hippocampal slices from mice were harvested with a vibratome (Leica VT1200S) $1 \mathrm{~h}$ after the mice were injected with peptide $(10 \mu \mathrm{m} / \mathrm{kg}$, i.p.). The slices were recovered in ACSF (in mM: $\mathrm{NaCl} 119, \mathrm{KCl} 2.5, \mathrm{NaHCO}_{3} 26.2, \mathrm{NaH}_{2} \mathrm{PO}_{4} 1$, glucose $11, \mathrm{MgCl}_{2} 1.3, \mathrm{CaCl}_{2} 2.5$ ) at $35^{\circ} \mathrm{C}$ for $30 \mathrm{~min}$ and then incubated at room temperature. After at least $1 \mathrm{~h}$ of recovery, the slices were transferred to a submerged recording chamber and perfused with ACSF at $4 \mathrm{ml} / \mathrm{min}$. Picrotoxin $(100 \mu \mathrm{M})$ was added to the ACSF during recording and a cut was made at $\mathrm{CA} 3$ to avoid epileptic responses. fEPSPs were recorded in the SC-CA1 synapse at 0.033 $\mathrm{Hz}$ for $30 \mathrm{~min}$ and $100 \mathrm{~Hz}, 1 \mathrm{sec}$ stimulation was used to induce LTP. The resistances of the recording electrodes were 1-2 M $\Omega$ and ACSF was used as the internal solution.

\section{Fear conditioning}

Before the fear conditioning experiment, mice were handled for 3 consecutive days. On the training day, mice were injected with $10 \mu \mathrm{m} / \mathrm{kg}$ peptide (i.p.) $1 \mathrm{~h}$ before the test, and transferred to a rectangular chamber capable of delivering foot-shocks (Med Associates). Three paired CS and US trials were carried out in the experiment. First, mice were allowed to freely explore the chamber for $3 \mathrm{~min}$ and were then exposed to a $30-\mathrm{sec}, 5000 \mathrm{~Hz}$ tone (CS). During the final $2 \mathrm{sec}$ of each CS, a $0.5-\mathrm{mA}$ foot-shock (US) was delivered. After the shock, a 90-sec inter-trial interval preceded the next identical trial. To evaluate contextual fear learning, $24 \mathrm{~h}$ after training the mice were placed in the chamber for $5 \mathrm{~min}$ in the absence of the CS or US and their freezing behavior was assessed. To evaluate cued fear conditioning, $48 \mathrm{~h}$ after the contextual fear test the mice were placed in a different chamber and exposed to the $\mathrm{CS}$ at $3 \mathrm{~min}$. Freezing behavior was assessed before and after presentation of the CS, and mice were removed from the chamber after a total of $5 \mathrm{~min}$.

\section{Protein extraction}

Non-denaturing membrane solubilization Neurons from cortical cultures were rinsed three times in ECS and extracted with pre-cooled lysis buffer (in $\mathrm{mM}$ : 50 Tris $\cdot \mathrm{HCl}, 0.1 \mathrm{PMSF}, 1$ aprotinin, $\mathrm{pH}$ 7.4). The neurons were then ultrasonicated twice for 8 sec each. Rat cortical tissue was first extracted in cold PBS and homogenized in lysis buffer (in mM: 50 Tris $\cdot \mathrm{HCl}, 320$ glucose, 0.1 
PMSF, 1 aprotinin, $\mathrm{pH} 7.4)$. Then 0.1 volume of Buffer A (10\% deoxycholate, $\mathrm{pH} 7.4$ ) was added to the prepared cell or tissue samples, and incubated at $36{ }^{\circ} \mathrm{C}$ for $0.5 \mathrm{~h}$. Next, 0.1 volume of Buffer B (500 mM Tris HCl, 1\% Triton X-100, pH 9.0) was added. All samples were dialyzed overnight in binding buffer $(50 \mathrm{mM}$ Tris. $\mathrm{HCl}, 0.1 \%$ Triton X-100, $\mathrm{pH} 7.4$ ), and centrifuged at $37000 \times$ $g$ for $30 \mathrm{~min}$ at $4{ }^{\circ} \mathrm{C}$. After centrifugation, the supernatant was used for co-immunoprecipitation experiments.

Denaturing membrane solubilization Cortical cultures were rinsed three times in ECS, lysed with RIPA buffer (50 mM Tris $\mathrm{HCl}, 150 \mathrm{mM} \mathrm{NaCl}, 1 \%$ Triton $\mathrm{X}-100,1 \%$ sodium deoxycholate, $0.1 \%$ SDS, $0.1 \mathrm{mM}$ PMSF, $1 \mathrm{mM}$ aprotinin, $\mathrm{pH} 7.4$ ), and the extracted neurons were ultrasonicated twice for $8 \mathrm{sec}$ each followed by incubation at $4{ }^{\circ} \mathrm{C}$ for $2 \mathrm{~h}$. Samples were then centrifuged at 12 $000 \times g$ for $10 \mathrm{~min}$ at $4{ }^{\circ} \mathrm{C}$ and the supernatant was used for SDSPAGE.

Rat brain tissue was homogenized in RIPA buffer (as above), centrifuged at $700 \times \mathrm{g}$ for $10 \mathrm{~min}$ at $4{ }^{\circ} \mathrm{C}$, the supernatant was incubated at $4{ }^{\circ} \mathrm{C}$ for $2 \mathrm{~h}$, and then centrifuged at $12000 \times \mathrm{g}$ for $10 \mathrm{~min}$ at $4{ }^{\circ} \mathrm{C}$; the supernatant was used for SDS-PAGE.

\section{Co-localization}

For co-localization, hippocampal neurons on slides were fixed with $4 \%$ paraformaldehyde for 10 min after a brief rinse in ECS, and then permeabilized and blocked simultaneously in PBS containing $0.5 \%$ Triton X-100 and 5\% BSA for $0.5 \mathrm{~h}$. Then the neurons were incubated in PBS containing both primary antibodies to GFP (rabbit anti-GFP) and Bip (mouse anti-Bip), or GFP (rabbit anti-GFP) and PSD95 (mouse anti-PSD95), or GluA1 (rabbit anti-GluA1) and PSD95 (mouse anti-PSD95) in PBS containing $0.5 \%$ Triton $\mathrm{X}-100$ for $1 \mathrm{~h}$, and after rinsing in PBS, neurons were incubated with PBS containing both Alexa 488-conjugated anti-rabbit secondary antibody and Alexa 546-conjugated anti-mouse secondary antibody. After a brief rinse, neurons were examined under a $60 \times, 1.4$ numerical aperture oil-immersion objective on an Olympus confocal microscope, and co-localization ratios were calculated using ImageJ software.

\section{Co-immunoprecipitation}

The protein concentration of the supernatant was adjusted to 1 $\mu \mathrm{g} / \mu \mathrm{l}$. The corresponding antibody was added to $200 \mu \mathrm{l}$ of sample and incubated overnight at $4{ }^{\circ} \mathrm{C}$. Then $30 \mu \mathrm{l}$ of solubilized protein A-sepharose beads was added to incubate for another $2 \mathrm{~h}$. The sample was rinsed three times with binding buffer and once with binding buffer containing $500 \mathrm{mM} \mathrm{NaCl}$ to remove non-specific interactions. After final centrifugation, the sample was incubated with $2 \times$ sample buffer and incubated at $90{ }^{\circ} \mathrm{C}$ for $10 \mathrm{~min}$, then used for SDS-PAGE. For input, $15 \mu 1$ of $4 \times$ sample buffer was added to $45 \mu 1$ of sample and then incubated at $90{ }^{\circ} \mathrm{C}$ for $10 \mathrm{~min}$ before being used for western blot.

\section{Antibodies and reagents}

The primary antibodies used were goat anti-GluN1, mouse anti-PDS-95 (ab13552, Abcam), anti-Bip (ab151269, Abcam), anti-GAPDH (92590, Millipore), anti-tau (ab28031, Abcam), rabbit anti-GluN2A (5546-1, Abcam), anti-Bip (ab21685, Abcam), anti-GRP94 (ab3674, Abcam) and anti-GFP (Ab290, Abcam). The secondary antibodies were goat anti-rabbit IgG-HRP (31420,
Pierce), goat anti-mouse IgG-HRP (31460, Pierce), donkey antigoat-HRP (31400, Pierce), donkey anti-rabbit IgG-Alexa 546 (a10040, Invitrogen), donkey anti-mouse IgG-Alexa 488 (a21202, Invitrogen), donkey anti-rabbit-Alexa 488 (a21206, Invitrogen) and donkey anti-mouse IgG-Alexa 546 (a11001, Invitrogen). The reagents used were BFA (B6542, Sigma), CBM (180467, Sigma), cytochalasin D (C8273, Sigma) and nocodazole (M1404, Sigma).

\section{Statistical analysis}

All data were analyzed using GraphPad prism 5 software and are represented as mean $\pm \mathrm{SEM}$. Data from multiple groups were quantified using one-way ANOVA, and comparisons of two groups were quantified by the student's $t$-test.

\section{Acknowledgments}

This work was supported by grants from the National Basic Research Program of China (2010CB912002), the National Natural Science Foundation of China (91232303, 81221003, 81371302 and 31460258) and the Fundamental Research Funds for the Central Universities of China. We thank Dr Guoqiang Bi from the University of Science and Technology of China for his help with STORM and other cell imaging experiments.

\section{References}

1 Cull-Candy S, Brickley S, Farrant M. NMDA receptor subunits: diversity, development and disease. Curr Opin Neurobiol 2001; 11:327-335.

2 Yashiro K, Philpot BD. Regulation of NMDA receptor subunit expression and its implications for LTD, LTP, and metaplasticity. Neuropharmacology 2008; 55:1081-1094.

3 de Armentia ML, Sah P. Development and subunit composition of synaptic NMDA receptors in the amygdala: NR2B synapses in the adult central amygdala. J Neurosci 2003; 23:6876-6883.

4 Nase G, Weishaupt J, Stern P, Singer W, Monyer H. Genetic and epigenetic regulation of NMDA receptor expression in the rat visual cortex. Eur J Neurosci 1999; 11:4320-4326.

5 Liu XB, Murray KD, Jones EG. Switching of NMDA receptor $2 \mathrm{~A}$ and $2 \mathrm{~B}$ subunits at thalamic and cortical synapses during early postnatal development. J Neurosci 2004; 24:8885-8895.

6 Philpot BD, Espinosa JS, Bear MF. Evidence for altered NMDA receptor function as a basis for metaplasticity in visual cortex. J Neurosci 2003; 23:5583-5588.

7 Sawtell NB, Frenkel MY, Philpot BD, Nakazawa K, Tonegawa S, Bear MF. NMDA receptor-dependent ocular dominance plasticity in adult visual cortex. Neuron 2003; 38:977-985.

8 Yashiro K, Corlew R, Philpot BD. Visual deprivation modifies both presynaptic glutamate release and the composition of perisynaptic/extrasynaptic NMDA receptors in adult visual cortex. J Neurosci 2005; 25:11684-11692.

9 Philpot BD, Sekhar AK, Shouval HZ, Bear MF. Visual experience and deprivation bidirectionally modify the composition and function of NMDA receptors in visual cortex. Neuron 2001; 29:157-169.

10 Philpot BD, Cho KKA, Bear MF. Obligatory role of NR2A for metaplasticity in visual cortex. Neuron 2007; 53:495-502. 
11 Quinlan EM, Lebel D, Brosh I, Barkai E. A molecular mechanism for stabilization of learning-induced synaptic modifications. Neuron; 41:185-192.

12 Kopp C, Longordo F, Lüthi A. Experience-dependent changes in NMDA receptor composition at mature central synapses. Neuropharmacology 2007; 53:1-9.

13 Chen B-S, Roche KW. Regulation of NMDA receptors by phosphorylation. Neuropharmacology 2007; 53:362-368.

14 Chung HJ, Huang YH, Lau LF, Huganir RL. Regulation of the NMDA receptor complex and trafficking by activity-dependent phosphorylation of the NR2B subunit PDZ ligand. $J$ Neurosci 2004; 24:10248-10259.

15 Tovar KR, Westbrook GL. Mobile NMDA receptors at hippocampal synapses. Neuron 2002; 34:253-264.

16 Groc L, Heine M, Cousins SL, et al. NMDA receptor surface mobility depends on NR2A-2B subunits. Proc Natl Acad Sci USA 2006; 103:18769-18774.

17 Prybylowski K, Chang K, Sans N, Kan L, Vicini S, Wenthold RJ. The synaptic localization of NR2B-containing NMDA receptors is controlled by interactions with PDZ proteins and AP-2. Neuron 2005; 47:845-857.

18 Vissel B, Krupp JJ, Heinemann SF, Westbrook GL. A use-dependent tyrosine dephosphorylation of NMDA receptors is independent of ion flux. Nat Neurosci 2001; 4:587-596.

19 Horton AC, Ehlers MD. Dual modes of endoplasmic reticulum-to-Golgi transport in dendrites revealed by live-cell imaging. J Neurosci 2003; 23:6188-6199.

20 Ramirez OA, Couve A. The endoplasmic reticulum and protein trafficking in dendrites and axons. Trends Cell Biol 2011; 21:219-227.

21 Guillaud L, Setou M, Hirokawa N. KIF17 dynamics and regulation of NR2B trafficking in hippocampal neurons. $J$ Neurosci 2003; 23:131-140.

22 Setou M, Nakagawa T, Seog DH, Hirokawa N. Kinesin superfamily motor protein KIF17 and mLin-10 in NMDA receptorcontaining vesicle transport. Science 2000; 288:1796-1802.

23 Jeyifous O, Lwaites C, Specht CG, et al. SAP97 and CASK mediate sorting of NMDA receptors through a previously unknown secretory pathway. Nat Neurosci 2009; 12:1011U1081.

24 Mauceri D, Gardoni F, Marcello E, Di Luca M. Dual role of CaMKII-dependent SAP97 phosphorylation in mediating trafficking and insertion of NMDA receptor subunit NR2A. $J$ Neurochem 2007; 100:1032-1046.

25 Swanger SA, He YA, Richter JD, Bassell GJ. Dendritic GluN2A synthesis mediates activity-induced NMDA receptor insertion. J Neurosci 2013; 33:8898-8908.

26 Udagawa T, Swanger SA, Takeuchi K, et al. Bidirectional control of mRNA translation and synaptic plasticity by the cytoplasmic polyadenylation complex. Mol Cell 2012; 47:253266.

27 Qiu S, Zhang XM, Cao JY, et al. An endoplasmic reticulum retention signal located in the extracellular amino-terminal domain of the NR2A subunit of N-Methyl-D-aspartate receptors. J Biol Chem 2009; 284:20285-20298.

28 Dani A, Huang B, Bergan J, Dulac C, Zhuang X. Superresolution imaging of chemical synapses in the brain. Neuron 2010; 68:843-856.

29 Hayashi Y, Shi SH, Esteban JA, Piccini A, Poncer JC, Ma- linow R. Driving AMPA receptors into synapses by LTP and CaMKII: requirement for GluR1 and PDZ domain interaction. Science 2000; 287:2262-2267.

30 Lu WY, Man HY, Ju W, Trimble WS, MacDonald JF, Wang YT. Activation of synaptic NMDA receptors induces membrane insertion of new AMPA receptors and LTP in cultured hippocampal neurons. Neuron 2001; 29:243-254.

31 Ori-McKenney Kassandra M, Jan LY, Jan YN. Golgi outposts shape dendrite morphology by functioning as sites of acentrosomal microtubule nucleation in neurons. Neuron 2012; 76:921-930.

32 Klausner RD, Donaldson JG, Lippincott-Schwartz J. Brefel$\operatorname{din} \mathrm{A}$ : insights into the control of membrane traffic and organelle structure. J Cell Biol 1992; 116:1071-1080.

33 Hirokawa N, Niwa S, Tanaka Y. Molecular motors in neurons: transport mechanisms and roles in brain function, development, and disease. Neuron 2010; 68:610-638.

34 Torre ER, Steward O. Protein synthesis within dendrites: Glycosylation of newly synthesized proteins in dendrites of hippocampal neurons in culture. J Neurosci 1996; 16:5967-5978.

35 Horton AC, Racz B, Monson EE, Lin AL, Weinberg RJ, Ehlers MD. Polarized secretory trafficking directs cargo for asymmetric dendrite growth and morphogenesis. Neuron 2005; 48:757-771.

36 Pierce JP, Mayer T, McCarthy JB. Evidence for a satellite secretory pathway in neuronal dendritic spines. Curr Biol 2001; 11:351-355.

37 Biermann B, Ivankova-Susankova K, Bradaia A, et al. The sushi domains of GABAB receptors function as axonal targeting signals. J Neurosci 2010; 30:1385-1394.

38 Ramirez OA, Vidal RL, Tello JA, et al. Dendritic assembly of heteromeric $\gamma$-aminobutyric acid type B receptor subunits in hippocampal neurons. J Biol Chem 2009; 284:13077-13085.

39 Hasdemir B, Fitzgerald DJ, Prior IA, Tepikin AV, Burgoyne $\mathrm{RD}$. Traffic of $\mathrm{Kv} 4 \mathrm{~K}+$ channels mediated by KChIP1 is via a novel post-ER vesicular pathway. J Cell Biol 2005; 171:459469.

$40 \mathrm{Hu}$ T, Kao C-Y, Hudson RT, Chen A, Draper RK. Inhibition of secretion by 1,3-cyclohexanebis(methylamine), a dibasic compound that interferes with coatomer function. Mol Biol Cell 1999; 10:921-933.

41 Bellone C, Nicoll RA. Rapid bidirectional switching of synaptic NMDA receptors. Neuron 2007; 55:779-785.

42 Matta JA, Ashby MC, Sanz-Clemente A, Roche KW, Isaac JTR. mGluR5 and NMDA receptors drive the experience- and activity-dependent NMDA receptor NR2B to NR2A subunit switch. Neuron 2011; 70:339-351.

43 Kennedy MJ, Ehlers MD. Organelles and trafficking machinery for postsynaptic plasticity. Annu Rev Neurosci 2006; 29:325-362.

44 Correia SS, Bassani S, Brown TC, et al. Motor protein-dependent transport of AMPA receptors into spines during longterm potentiation. Nat Neurosci 2008; 11:457-466.

45 Zakharenko S, Chang S, O’Donoghue M, Popov SV. Neurotransmitter secretion along growing nerve processes: comparison with synaptic vesicle exocytosis. J Cell Biol 1999; 144:507-518.

46 Tanaka T, Serneo FF, Higgins C, Gambello MJ, Wynshaw-Boris A, Gleeson JG. Lis1 and doublecortin function with dynein 
to mediate coupling of the nucleus to the centrosome in neuronal migration. $J$ Cell Biol 2004; 165:709-721.

47 Sharp DJ, Yu W, Ferhat L, Kuriyama R, Rueger DC, Baas $\mathrm{PW}$. Identification of a microtubule-associated motor protein essential for dendritic differentiation. J Cell Biol 1997; 138:833-843.

$48 \mathrm{Xu}$ Z, Chen RQ, Gu QH, et al. Metaplastic regulation of longterm potentiation/long-term depression threshold by activity-dependent changes of NR2A/NR2B ratio. J Neurosci 2009; 29:8764-8773.

49 Sanz-Clemente A, Matta JA, Isaac JTR, Roche KW. Casein kinase 2 regulates the NR2 subunit composition of synaptic NMDA receptors. Neuron 2010; 67:984-996.

50 Chung HJ, Huang YH, Lau L-F, Huganir RL. Regulation of the NMDA receptor complex and trafficking by activity-dependent phosphorylation of the NR2B subunit PDZ ligand. $J$ Neurosci 2004; 24:10248-10259.

51 Zhang S, Edelmann L, Liu J, Crandall JE, Morabito MA. Cdk5 Regulates the phosphorylation of tyrosine 1472 NR2B and the surface expression of NMDA receptors. $J$ Neurosci 2008; 28:415-424.

52 Tovar KR, Westbrook GL. Mobile NMDA receptors at hippocampal synapses. Neuron 2002; 34:255-264.

53 Bramham CR, Wells DG. Dendritic mRNA: transport, translation and function. Nat Rev Neurosci 2007; 8:776-789.

54 Hanus C, Ehlers MD. Secretory outposts for the local processing of membrane cargo in neuronal dendrites. Traffic 2008; 9:1437-1445

55 Cui-Wang T, Hanus C, Cui T, et al. Local zones of endoplasmic reticulum complexity confine cargo in neuronal dendrites. Cell 2012; 148:309-321.

56 Park M, Penick EC, Edwards JG, Kauer JA, Ehlers MD. Recycling endosomes supply AMPA receptors for LTP. Science 2004; 305:1972-1975.

57 Ellgaard L, Helenius A. Quality control in the endoplasmic reticulum. Nat Rev Mol Cell Biol 2003; 4:181-191.

58 Otero JH, Lizák B, Hendershot LM. Life and death of a BiP substrate. Semin Cell Dev Biol 2010; 21:472-478.

59 Lee YK, Brewer JW, Hellman R, Hendershot LM. BiP and immunoglobulin light chain cooperate to control the folding of heavy chain and ensure the fidelity of immunoglobulin assembly. Mol Biol Cell 1999; 10:2209-2219.

60 Vanhove M, Usherwood YK, Hendershot LM. Unassembled Ig heavy chains do not cycle from $\mathrm{BiP}$ in vivo but require light chains to trigger their release. Immunity 2001; 15:105-114.

61 Pabba M, Wong AY, Ahlskog N, et al. NMDA receptors are upregulated and trafficked to the plasma membrane after sigma-1 receptor activation in the rat hippocampus. $J$ Neurosci 2014; 34:11325-11338.

62 Su TP, Hayashi T, Maurice T, Buch S, Ruoho AE. The sigma-1 receptor chaperone as an inter-organelle signaling mod- ulator. Trends Pharmacol Sci 2010; 31:557-566.

63 Ortega-Roldan JL, Ossa F, Schnell JR. Characterization of the human sigma-1 receptor chaperone domain structure and binding immunoglobulin protein (BiP) interactions. $J$ Biol Chem 2013; 288:21448-21457.

64 Peng Y, Zhao J, Gu QH, et al. Distinct trafficking and expression mechanisms underlie LTP and LTD of NMDA receptor-mediated synaptic responses. Hippocampus 2010; 20:646658.

65 Grosshans DR, Clayton DA, Coultrap SJ, Browning MD. LTP leads to rapid surface expression of NMDA but not AMPA receptors in adult rat CA1. Nat Neurosci 2002; 5:27-33.

66 Hunt DL, Castillo PE. Synaptic plasticity of NMDA receptors: mechanisms and functional implications. Curr Opin Neurobiol 2012; 22:496-508.

67 Thiagarajan TC, Lindskog M, Tsien RW. Adaptation to synaptic inactivity in hippocampal neurons. Neuron 2005; 47:725737.

68 Abraham WC. Metaplasticity: tuning synapses and networks for plasticity. Nat Rev Neurosci 2008; 9:387-387.

69 Kiyama Y, Manabe T, Sakimura K, Kawakami F, Mori H, Mishina M. Increased thresholds for long-term potentiation and contextual learning in mice lacking the NMDA-type glutamate receptor $\varepsilon 1$ subunit. J Neurosci 1998; 18:6704-6712.

70 von Engelhardt J, Doganci B, Jensen V, et al. Contribution of hippocampal and extra-hippocampal NR2B-containing NMDA receptors to performance on spatial learning tasks. Neuron 2008; 60:846-860.

71 Miserendino MJD, Sananes CB, Melia KR, Davis M. Blocking of acquisition but not expression of conditioned fear-potentiated startle by NMDA antagonists in amygdala. Nature 1990; 345:716-718.

72 Chenard BL, Menniti FS. Antagonists selective for NMDA receptors containing the NR2B subunit. Curr Pharm Des 1999; 5:381-404.

73 Rodrigues SM, Schafe GE, LeDoux JE. Intra-amygdala blockade of the NR2B subunit of the NMDA receptor disrupts the acquisition but not the expression of fear conditioning. $J$ Neurosci 2001; 21:6889-6896.

74 Qiu S, Zhang XM, Cao JY, et al. An endoplasmic reticulum retention signal located in the extracellular amino-terminal domain of the NR2A subunit of N-methyl-D-aspartate receptors. J Biol Chem 2009; 284:20285-20298.

75 Qiu S, Hua YL, Yang F, Chen YZ, Luo JH. Subunit assembly of N-methyl-D-aspartate receptors analyzed by fluorescence resonance energy transfer. J Biol Chem 2005; 280:2492324930.

76 Luo JH, Fu ZY, Losi G, et al. Functional expression of distinct NMDA channel subunits tagged with green fluorescent protein in hippocampal neurons in culture. Neuropharmacology 2002; 42:306-318.

(Supplementary information is linked to the online version of the paper on the Cell Research website.) 